\title{
Schisandra henryi C. B. Clarke in vitro cultures: a promising tool for the production of lignans and phenolic compounds
}

\author{
Karolina Jafernik ${ }^{1} \cdot$ Agnieszka Szopa $^{1} \mathbb{D} \cdot$ Magda Barnaś $^{1} \cdot$ Michał Dziurka $^{2} \cdot$ Halina Ekiert $^{1}$
}

Received: 26 March 2020 / Accepted: 21 July 2020 / Published online: 3 August 2020

(c) The Author(s) 2020

\begin{abstract}
We initiated and optimized in vitro culture conditions of the endemic Chinese plant species-Schisandra henryi C. B. Clarke. Different types of in vitro solid cultures (microshoot and callus), cultivation periods (10, 20, and 30 days), and selected concentrations of BA, IBA, $\mathrm{GA}_{3}(0$ to $3 \mathrm{mg} / \mathrm{l})$ in the Murashige and Skoog (MS) medium were tested. The presence of dibenzocyclooctadiene lignans (schisandrin, gomisin G, schisantherin A and B, deoxyschisandrin and schisandrin C), dibenzylbutane lignans (hernicine B), aryltetralin lignans (wulignan A1 and A2, epiwulignan A1, enshicine, epienshicine and dimethylwulignan A1), and triterpenoids: kadsuric acid and schisanhenric acid was confirmed by UHPLC-MS/MS analysis. Using HPLC-DAD, the qualitative and quantitative profiles of dibenzocyclooctadiene lignans, phenolic acids and flavonoids in methanolic extracts from biomass were estimated. The maximum total amounts of these groups of metabolites were $873.71,840.89$ and $421.98 \mathrm{mg} / 100 \mathrm{~g} \mathrm{DW}$, respectively. The main compounds were: schisantherin B (max. $622.59 \mathrm{mg} / 100 \mathrm{~g}$ DW), schisantherin A (max. $143.74 \mathrm{mg} / 100 \mathrm{~g}$ DW), neochlorogenic acid (max. $472.82 \mathrm{mg} / 100 \mathrm{~g} \mathrm{DW}$ ), caftaric acid (max. $370.81 \mathrm{mg} / 100 \mathrm{~g} \mathrm{DW}$ ), trifolin (max. $138.56 \mathrm{mg} / 100 \mathrm{~g} \mathrm{DW}$ ) and quercitrin (max. $122.54 \mathrm{mg} / 100 \mathrm{~g} \mathrm{DW}$ ). The highest total amounts of secondary metabolites estimated in the extracts from in vitro cultures were, respectively, 13.0, 7.0, and 1.4 times higher than in the leaf extracts analyzed for comparison. This is the first report on the biosynthetic potential of cells from Schisandra henryi in vitro cultures.
\end{abstract}

\section{Key message}

The initiation, broad optimization and comprehensive phytochemical studies of lignans, phenolic acids and flavonoids in the extracts from Schisandra henryi microshoot and callus in vitro cultures were elaborated for the first time.

Keywords Schisandra lignans $\cdot$ Phenolic acids $\cdot$ Flavonoids $\cdot$ Shoot-differentiating culture $\cdot$ Undifferentiating culture HPLC-DAD · UHPLC-MS/MS

\section{Introduction}

Communicated by Amita Bhattacharya.

Electronic supplementary material The online version of this article (https://doi.org/10.1007/s11240-020-01895-2) contains supplementary material, which is available to authorized users.

Agnieszka Szopa

a.szopa@uj.edu.pl

1 Chair and Department of Pharmaceutical Botany, Medical College, Jagiellonian University, Medyczna 9, 30-688 Kraków, Poland

2 The Franciszek Górski Institute of Plant Physiology, Polish Academy of Sciences, ul. Niezapominajek 21, 30-239 Kraków, Poland
Schisandra henryi C. B. Clarke (yi geng wu wei zi)Schisandraceae-is an endemic plant species native to the Yunnan Province of China (Saunders 2000; Szopa et al. 2019a). The medicinal properties of fruit extracts of this species are widely known in the traditional Chinese medicine (TCM) (Chen et al. 2010).

The most famous medicinal representative of the Schisandraceae family is Schisandra chinensis, which occupies an important position in the official East-Asian medicine, but also in North American and European phytomedicine (World Health Organization 2007; European Directorate for the Quality of Medicines 2008; Upton et al. 2011; European Directorate for the Quality of Medicines 2017). 
Taking into account this position of S. chinensis in world medicine and chemotaxonomical indications, we deemed it interesting to establish in vitro cultures of $S$. henryi and evaluate their biosynthetic possibilities.

In different types of in vitro cultures of $S$. chinensis and its cultivar-S. chinensis cv. Sadova we have previously found high amounts of bioactive dibenzocyclooctadiene lignans ("Schisandra chinensis lignans") and, additionally, other groups of polyphenols_- phenolic acids and flavonoids (Chen et al. 2020; Szopa et al. 2016, 2017b, 2018b, 2020; Liu et al. 2020). These three groups of metabolites are the most valuable antioxidants, also known because of their anticancer, immunostimulant, anti-inflammatory, hepatoregenerative, cardioprotective, anticoagulant, antimicrobial, and other valuable biological activities (Nijveldt et al. 2001; Heleno et al. 2015; Szopa et al. 2017a; He et al. 2020).

According to the available literature, the chemical composition of $S$. henryi is partly similar to that of $S$. chinensis, being dominated by dibenzocyclooctadiene lignans (Szopa et al. 2019a). The $S$. henryi dibenzocyclooctadiene lignans include: schisantherin A and schisantherin B, deoxyschisandrin, epischisandron, schisandrin, gomisin $\mathrm{G}$ and benzoylgomisin Q (Chen et al. 2005; Iu et al. 2009). Importantly, extracts of $S$. henryi fruit also contain other compounds from the group of tetrahydrofuran and aryltetralin lignans, which have been confirmed in shoot and leaf extracts (Liu et al. 1988; Li et al. 2004; Iu et al. 2009; Christophe 2013). The tetrahydrofuran lignans estimated in stems include ganschisandrin (Jian-min et al. 1989), and, identified as unique to this species, dibenzylbutane lignans: hernicine A and B (Iu et al. 2009). The group of aryltetralin lignans includes compounds that have been identified in stems-wulignan A1, wulignan A2, dimethylwulignan A1 and epiwulignan A (Liu et al. 1988), and in shoots-enshicine and epienshicine (Liu et al. 1988; Chen et al. 2003a). The second characteristic group of secondary metabolites found in $S$. henryi are triterpenoids (Chen et al. 2003a, 2010; Li et al. 2004).

Regarding the biological properties of $S$. henryi, they relate only to the cytotoxic activity of this plant species as the source of medicinal raw material (Chen et al. 2005; Wiart 2013).

Plant biotechnology creates valuable potential alternatives for the in vitro study of valuable, medicinal plants (Hammond et al. 2000; Verpoorte et al. 2002). However, there are no publications describing biotechnological studies with $S$. henryi, making the subject of our work relevant and topical.

The major purpose of this research work is to gain new knowledge on the initiation, optimization, and metabolomic profile of $S$. henryi in vitro cultures. Thus, we tested different types of agar cultures with regard to the degree of differentiation-microshoot and callus, different durations of growth periods: 10, 20, 30 days, and different concentrations of plant growth regulators (PGRs): BA (6-benzyladenine), IBA (indole-3-butyric acid), and $\mathrm{GA}_{3}$ (gibberellic acid), in concentrations from 0 to $3 \mathrm{mg} / \mathrm{l}$, in the Murashige and Skoog (MS) medium (Murashige and Skoog 1962). With the use of UHPLC-MS/MS and HPLC-DAD methods, we estimated the qualitative and quantitative metabolomic profiles of lignans, triterpenoids, phenolic acids and flavonoids in extracts from biomass from in vitro cultures, as well as in extracts from leaves of the parent plant.

\section{Materials and methods}

\section{Parent plant material}

Parent plant materials were obtained from the company "Clematis" (Pruszków, Poland) (https://www.clematis. com.pl/), who provided leaves of $S$. henryi male specimens for in vitro cultures, that were identified by Dr Szczepan Marczyński (the owner of Clematis arboretum). Leaves from a $S$. henryi parent plant were harvested in May and September of 2016 , and were air-dried at $25-30{ }^{\circ} \mathrm{C}$.

\section{Initiation of in vitro cultures}

Leaf buds to initiate in vitro cultures were collected in the spring of 2016 from a male specimen of Schisandra henryi C. B. Clarke from "Clematis" (Pruszków, Poland) arboeretum. They were degreased with $70 \%$ ethanol (30 s) and then subjected to further sterilization with $\mathrm{HgCl}_{2}$ (mercury chloride II) at $0.1 \%$ for $7 \mathrm{~min}$. Sterile buds were rinsed with sterile redistilled water and transferred to Murashige and Skoog (1962; MS) agar medium enriched with $1 \mathrm{mg} / \mathrm{l} \mathrm{BA}$ (6-benzyladenine) and $0.5 \mathrm{mg} / 1$ NAA (1-naphthaleneacetic acid) as PGRs (Bhojwani and Razdan 1989; Mohan 2016).

\section{Experimental microshoot and callus cultures}

The study involved cultivation of agar microshoot and callus cultures on MS medium with 3\% (w/v) sucrose and $0.72 \%$ (w/v) agar (plant agar, Duchefa). Cultures were maintained at $25 \pm 2{ }^{\circ} \mathrm{C}$, under continuous artificial illumination of LED white light, with a photosynthetic photon flux density (PPFD) of $40 \mu \mathrm{mol} \mathrm{m} \mathrm{m}^{-2} \mathrm{~s}^{-1}$, and subcultured at 30-day intervals.

Durations of 10-, 20- and 30-day growth cycles (3 series) were tested for both types of cultures. The growth index (Gi) was calculated on the basis of weight of dry tissue according to formula: $\mathrm{Gi}=\left(\mathrm{DW}_{1}-\mathrm{DW}_{0}\right) / \mathrm{DW}_{0}$ : where $\mathrm{DW}_{0}$ was the weight of inoculum and $\mathrm{DW}_{1}$ the final weight of tissue after culture growth period.

Agar microshoot cultures were grown in plant tissuededicated glass containers (Sigma-Aldrich No. V8630). For 
the experiment, $0.5 \mathrm{~g}$ of inoculum per vessel was used. For microshoot cultures, media differed in the concentration of $\mathrm{BA}, \mathrm{IBA}$ (indole-3-butyric acid), and $\mathrm{GA}_{3}$ (gibberellic acid) (mg/l): 1,1 and 0 (variant A), 1, 1 and 0.25 (variant B), 0.25, 2 and 0 (variant $C$ ), 0.25, 3 and 0 (variant $\mathrm{D}$ ), 2, 1 and 0 (variant E), 0.5, 2 and 0 (variant F), respectively. For callus cultures, a single MS medium was tested with $1 \mathrm{mg} / \mathrm{l} \mathrm{BA}$ and $1 \mathrm{mg} / \mathrm{l}$ IBA (i.e. variant A), as callus cultures were proved to contain much lower amounts of the lignans estimated (Bhojwani and Razdan 1989; Mohan 2016).

\section{Chromatographic analyses}

To prepare methanolic extracts, samples of dry biomass powder were weighed as follows: $0.2 \mathrm{~g}$ each (harvested after 10 days - microshoot cultures), $0.3 \mathrm{~g}$ (harvested after 20 and 30 days-microshoot cultures) and $1 \mathrm{~g}$ (harvested after 10 , 20 and 30 days-callus cultures) (3 samples in 3 replications). The material was subjected to extraction with methanol ( $5 \mathrm{ml}$-microshoot cultures, $8 \mathrm{ml}$-callus cultures collected after 10 days, $10 \mathrm{ml}$-callus cultures collected after 20 days, and $8 \mathrm{ml}$-callus cultures collected after 30 days) (purity for HPLC analysis by Merck, Germany). The extraction was carried out in an ultrasonic bath (Sonic-2, POLSONIC, Poland; ultrasonic power $2 \times 100 \mathrm{~W}, 40 \mathrm{kHz}$, water bath dimensions $150 \times 135 \times 100 \mathrm{~mm}, 1.61$ volume) twice for $30 \mathrm{~min}$. The extracts were centrifuged in a centrifuge (MPW Med. Instruments, Poland, model-Centrifuge MPW-223E) at $4000 \mathrm{rpm}$ for $5 \mathrm{~min}$. The extracts were then filtered through sterile syringe filters (Millex ${ }^{\circledR} G P$, Millipore, Merck, Germany, $0.22 \mu \mathrm{m}$ Filter Unit) into vials for HPLC (Witko Sp. z.o.o., Poland) (Harborne 1984; Mohan 2016).

Qualitative analyses of lignans and triterpenenoids were performed using a UHPLC-MS/MS tandem mass spectrometer with a triple quadrupole (QQQ) mass filter (Agilent $6410 \mathrm{LC} / \mathrm{MS}$ ), coupled with an ultra-high-performance chromatograph (Agilent 1260). Samples were separated on an analytical column (Kinetex C18 $150 \times 4.6 \mathrm{~mm}, 2.6 \mu \mathrm{m}$ ) in a gradient mode of $50 \%$ methanol in water (A) versus $100 \%$ methanol (B) with $0.1 \%$ of formic acid. A linear gradient was applied, $20 \%$ to $65 \%$ of $\mathrm{B}$ in $22 \mathrm{~min}$. at $0.5 \mathrm{ml} / \mathrm{min}$. at $60{ }^{\circ} \mathrm{C}$; the injection volume was $2 \mu \mathrm{l}$. Analyses were conducted in positive ionization (+ ESI), drying gas temperature was $350{ }^{\circ} \mathrm{C}$, gas flow at $12 \mathrm{l} / \mathrm{min}$., and nebulizer pressure at 35 psi. Standard lignan substances were purchased from ChemFaces Biochemical Co. Ltd. (China). Qualitative and quantitative analyses of dibenzocyclooctadiene lignans were performed using high performance liquid chromatography with a DAD detector (HPLC-DAD), as we validated previously (Szopa et al. 2016, 2018a, b). Separation was conducted on a Kinetex ${ }^{\mathrm{TM}} \mathrm{C} 18(150 \times 4.6 \mathrm{~mm} ; 2.6 \mu \mathrm{m})$ column. The mobile phase consisted of A-acetonitrile, B-redistilled water, in gradient program. The flow rate was $0.8 \mathrm{ml} /$ min., and column temperature was set at $30{ }^{\circ} \mathrm{C}$. The injection volume was $5 \mu \mathrm{l}$ and the compounds of interest were detected at $225 \mathrm{~nm}$. Identification and quantification were performed by comparison with standards, based on retention times and calibration curves. The following standards of lignans were applied: schisandrin, gomisin A, gomisin G, schisantherin A, schisantherin B, schisanhenol, deoxyschisandrin, $\gamma$-schisandrin, schisandrin $C$.

Qualitative and quantitative analyses of phenolic acids and flavonoids in the methanolic extracts were performed by a validated method (Ellnain-Wojtaszek and Zgórka 1999; Sułkowska-Ziaja et al. 2017), using a Merck-Hitachi liquid chromatograph (LaChrom Elite) with a DAD L-2455 detector (HPLC-DAD). Separation was conducted on Purospher RP-18 $(250 \times 4 \mathrm{~mm} ; 5 \mu \mathrm{m}$, Merck, Germany) column. The mobile phase consisted of A-methanol, $0.5 \%$ acetic acid $1: 4$ and $\mathrm{B}-$ methanol $(\mathrm{v} / \mathrm{v})$, in gradient program. The flow rate was $1 \mathrm{ml} / \mathrm{min}$. and column temperature was set to $25^{\circ} \mathrm{C}$. The injection volume was $10 \mu \mathrm{l}$ and the compounds of interest were detected at $254 \mathrm{~nm}$. Identification and quantification were performed by comparison with standards, based on retention times and calibration curves. The following standards of phenolic acids and their precursors were applied: benzoic, 3,4-dihydroxyphenylacetic, ellagic, gallic, gentisic, p-hydroxybenzoic, protocatechuic, salicylic, syringic vanillic, cinnamic, caffeic, o-coumaric, m-coumaric, p-coumaric, ferulic, hydrocaffeic, isoferulic, sinapic, caftaric, chlorogenic, cryptochlorogenic, isochlorogenic, neochlorogenic and rosmarinic acids (HPLC purity $\geq 98.0 \%$, Sigma-Aldrich). The flavonoid standards included: vitexin, cynaroside, quercimitrin, rutoside, myricetin, hyperoside, quercitrin, apigetrin, trifolin, quercetin, luteolin, kaempferol, kaempferol-7-ramnoside (HPLC purity $\geq 95.0 \%$, SigmaAldrich, USA).

\section{Statistical analysis}

All data are presented as means \pm SD of four independent experiments and determinations. Asterisk (*) indicate significant differences between means (Student's test $\mathrm{n}=10$, $\mathrm{p}<0.05)$. Statistical analysis of the results was carried out using the Statistica 10 (StatSoft, USA) program.

\section{Results}

\section{Morphology and growth index}

Microshoots and callus tissue appeared from $S$. henryi leaf buds maintained on MS medium with $1 \mathrm{mg} / \mathrm{l} \mathrm{BA}$ and $1 \mathrm{mg} / \mathrm{l}$ IBA after 30 days (Fig. S1). All tested MS medium variants had a significant impact on $S$. henryi microshoot multiplication via axillary bud development (Table S1). 
The induction percentage of the new microshoots was relatively low (Table S1). The highest multiplication rate was obtained for the explants maintained on MS medium variant $\mathrm{A}(88 \%)$ and $\mathrm{F}(85 \%)$ (Table $\mathrm{S} 1)$, where microshoots were also longer ( 2.25 and $2.12 \mathrm{~cm}$, respectively) (Table S1).

The effects of PGRs (six MS medium variants: A-F for microshoots and A for callus cultures), and the duration of the growth cycle (three different cultivation periods-10, 20 and 30 days) on the growth and appearance of biomass were observed for both types of cultures (Fig. 1). The morphological appearance of microshoot cultures on the tested medium variants differed in each growth cycle. Microshoots growing on variants $\mathrm{A}$ and $B$ were characterized by a significant number of microshoots, dark green in colour and strongly spread (Fig. 1). The effect of growth cycle duration on the appearance of microshoot cultures was also observed-periods of 20 and 30 days were the most favourable. The growth index (Gi) varied from $38.28 \%$ to $75.58 \%$ (Fig. 2). The highest $\mathrm{Gi}$ value was obtained for microshoots cultivated for 30 days on variant A of MS medium (Fig. 2a).

The appearance of callus culture biomass did not depend on the duration of the culture cycle. The undifferentiated tissue was light green, but after 30 days of growth browning of the biomass was documented (Fig. 1). An increase in callus tissue was observed with the extension of the growth cycle. The growth index (Gi) varied from $82.55 \%$ to $91.34 \%$. The highest Gi value was obtained for callus cultivated for 30 days (Fig. 2b).

\section{UHPLC-MS/MS qualitative analyses}

The qualitative profiles of lignans from in vitro biomass extracts of all the tested cultures were the same. The UHPLC-MS/MS of methanolic extracts confirmed the presence of compounds from three groups of lignans and, additionally, two triterpenoid compounds were identified. The results of UHPLC-MS/MS analyses are summarized in Table S2. The presence of dibenzocyclooctadiene lignans (schisandrin, gomisin G, schisantherin A and B, deoxyschisandrin and schisandrin $C$ ), dibenzylbutane lignans (hernicine B) and aryltetralin lignans (wulignan A1 and A2, epiwulignan A1, enshicine, epienshicine and dimethylwulignan A1) was confirmed (Table S2) (Taguchi and Ikeya 1975; Ikeya et al. 1979a, b, 1980, 1982a, b, 1986, 1988, 1990; Jia-Sen et al. 1988; Liu et al. 1988; He et al. 1997; Deng et al. 2008; Wang et al. 2011; Yang et al. 2011). Moreover, kadsuric acid and schisanhenric acid, from the triterpendoids group, were confirmed by UHPLC-MS/MS analysis (Table S2) (Jia-Sen et al. 1980; Zou et al. 2015).

\section{Production of dibenzocyclooctadiene lignans}

\section{Microshoot cultures}

The effects of PGRs on lignan content were evident in agar-grown microshoots. In all extracts obtained from stationary cultures, 6 of the 9 tested dibenzocyclooctadiene lignans were detected-schisandrin, gomisin G, schisantherin A, schisantherin B, deoxyschisandrin and schisandrin C (Tables 1 and S2-S5). The amounts of individual

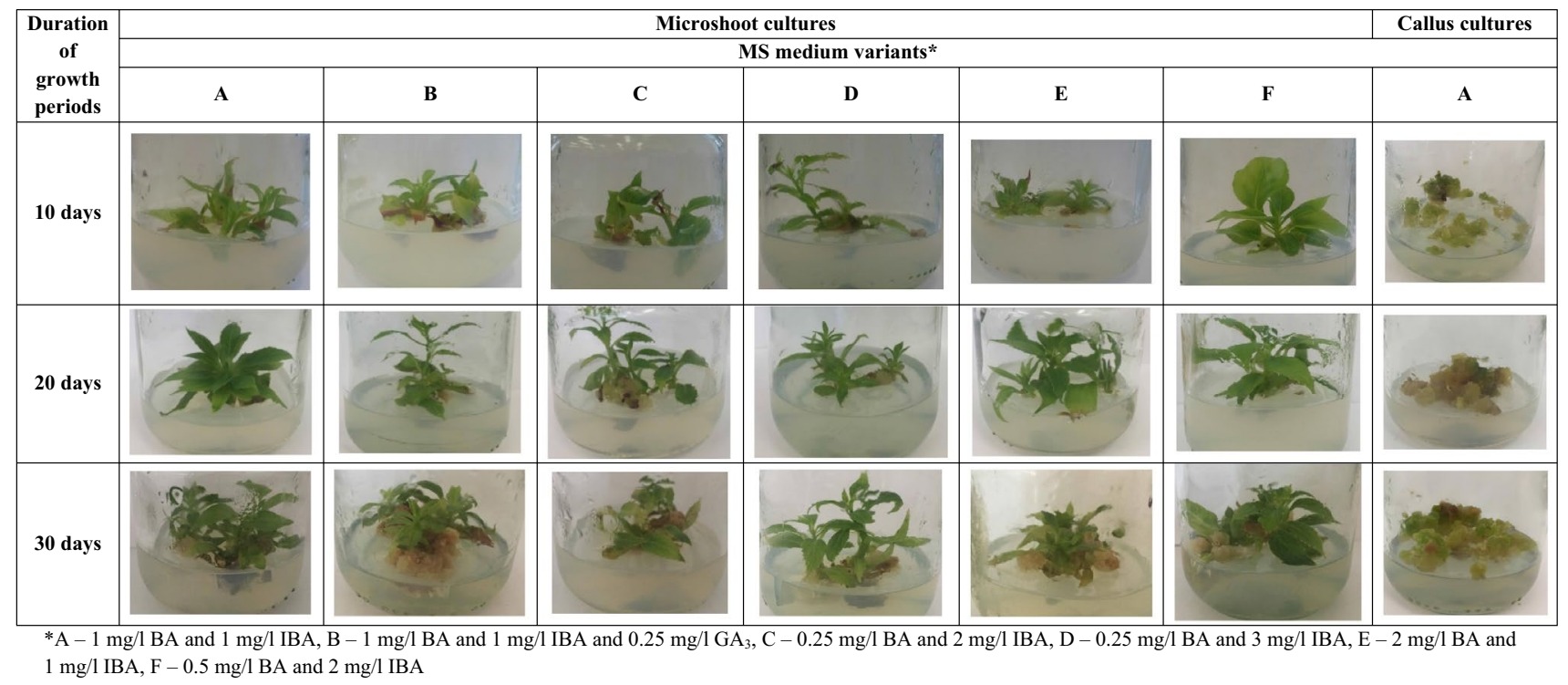

Fig. 1 Experimental S. henryi microshoot and callus cultures - the appearance of biomasses depending on the applied growth periods and the composition of PGR's used 
Fig. 2 The growth index factor (Gi) of dry biomasses of $S$. hen$r y i$, a agar microshoot cultures maintained on different MS medium variants*, and $\mathbf{b}$ callus cultures (a)

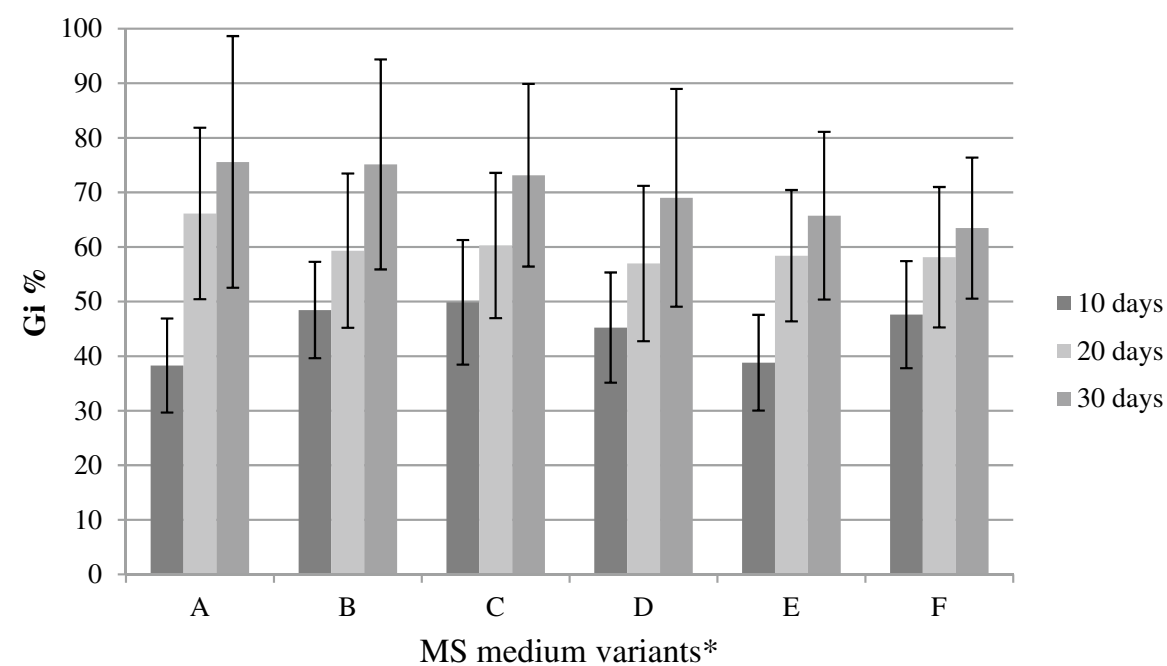

(b)

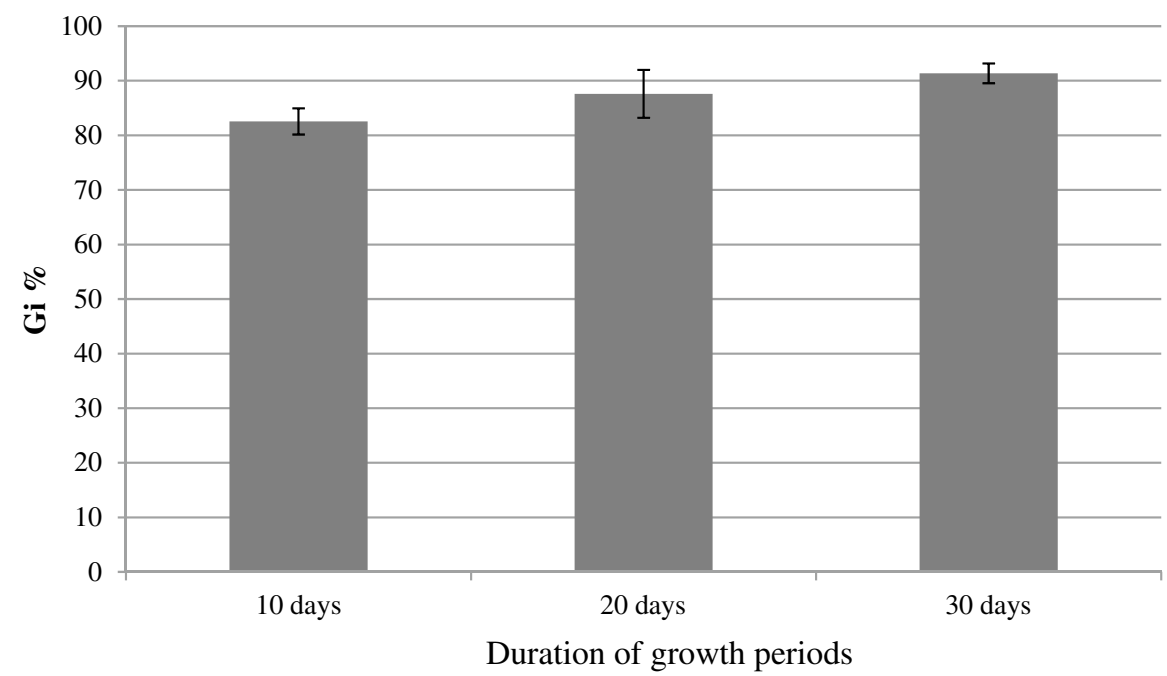

*A $-1 \mathrm{mg} / \mathrm{l} \mathrm{BA}$ and $1 \mathrm{mg} / \mathrm{l} \mathrm{IBA}, \mathrm{B}-1 \mathrm{mg} / \mathrm{l} \mathrm{BA}$ and $1 \mathrm{mg} / \mathrm{l} \mathrm{IBA}$ and $0.25 \mathrm{mg} / \mathrm{l} \mathrm{GA}, \mathrm{C}-0.25 \mathrm{mg} / \mathrm{l} \mathrm{BA}$ and 2 $\mathrm{mg} / \mathrm{l} \mathrm{IBA}, \mathrm{D}-0.25 \mathrm{mg} / \mathrm{l} \mathrm{BA}$ and $3 \mathrm{mg} / \mathrm{l} \mathrm{IBA}, \mathrm{E}-2 \mathrm{mg} / \mathrm{l} \mathrm{BA}$ and $1 \mathrm{mg} / \mathrm{l} \mathrm{IBA}, \mathrm{F}-0.5 \mathrm{mg} / \mathrm{l} \mathrm{BA}$ and $2 \mathrm{mg} / \mathrm{lBA}$ compounds varied dependent on the medium variant and duration of the growth period, ranging from $0.14 \mathrm{mg} / 100 \mathrm{~g}$ DW (deoxyschisandrin, MS variant C, 10 days) to $622.59 \mathrm{mg} / 100 \mathrm{~g} \mathrm{DW}$ (schisantherin B, MS variant F, 10 days). In all analyzed samples, the quantitatively dominant compounds were: schisandrin, schisantherin $\mathrm{A}$ and schisantherin B. Their amounts varied from $6.53 \mathrm{mg} / 100 \mathrm{~g}$ DW (MS variant B, 30 days) to $61.24 \mathrm{mg} / 100 \mathrm{~g}$ DW (MS variant $F, 10$ days), from $31.50 \mathrm{mg} / 100 \mathrm{~g}$ DW (MS variant $\mathrm{D}, 30$ days) to $143.74 \mathrm{mg} / 100 \mathrm{~g}$ DW (MS variant $\mathrm{F}, 10$ days), and from $140.21 \mathrm{mg} / 100 \mathrm{~g} \mathrm{DW}$ (MS variant D, 30 days) to $622.59 \mathrm{mg} / 100 \mathrm{~g}$ DW (variant $\mathrm{F}, 10$ days), respectively (Tables 1 and S3-S5, Fig. 3a).

The obtained total amounts of lignans also depended on the MS medium variant and applied duration of growth periods. The lowest total amount (213.78 mg/100 g DW) was confirmed for extracts from the biomass collected after 30 days of growth on variant D (Tables 1 and S5, Fig. S2a). The highest total amount of dibenzocyclooctadiene lignans $(873.71 \mathrm{mg} / 100 \mathrm{~g} \mathrm{DW})$ was recorded for extracts from microshoots cultivated on variant $\mathrm{F}$ of MS medium, harvested after 10 days of cultivation (Tables 1 and S3, Fig. S2a).

\section{Callus cultures}

In all extracts obtained from callus cultures, the same 6 of the 9 tested dibenzocyclooctadiene lignans as in microshoot cultures were detected (Tables 2 and S6). The amounts of individual compounds also depended on the 
(a)

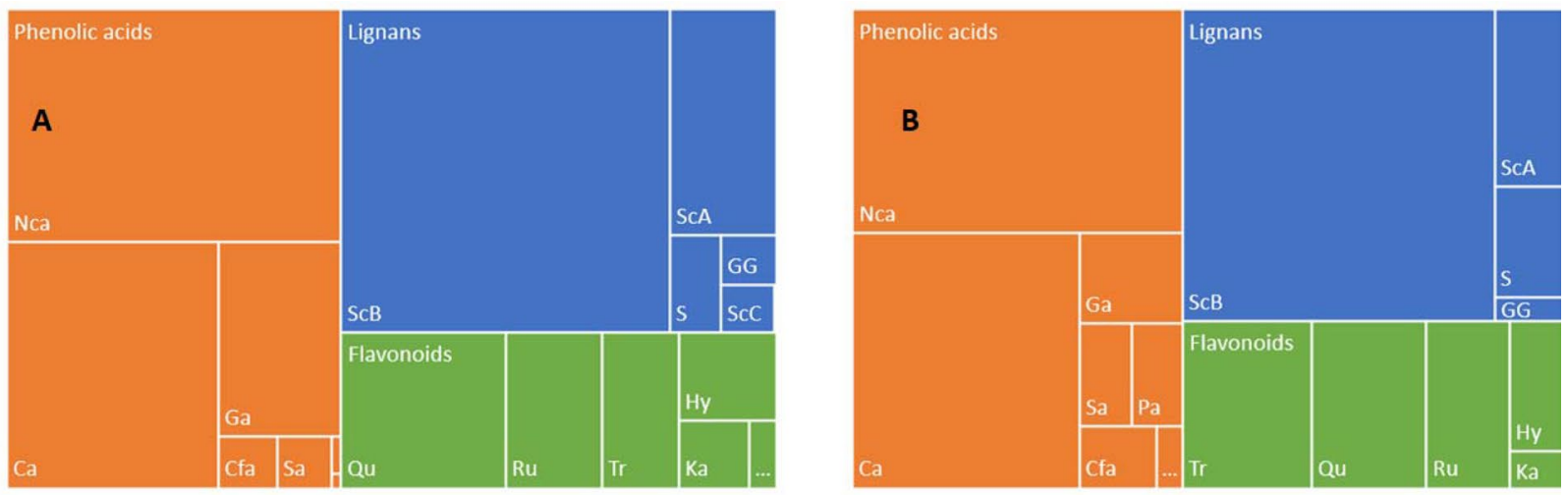

- Lignans $=$ Phenolic acids = Flavonoids

(b)
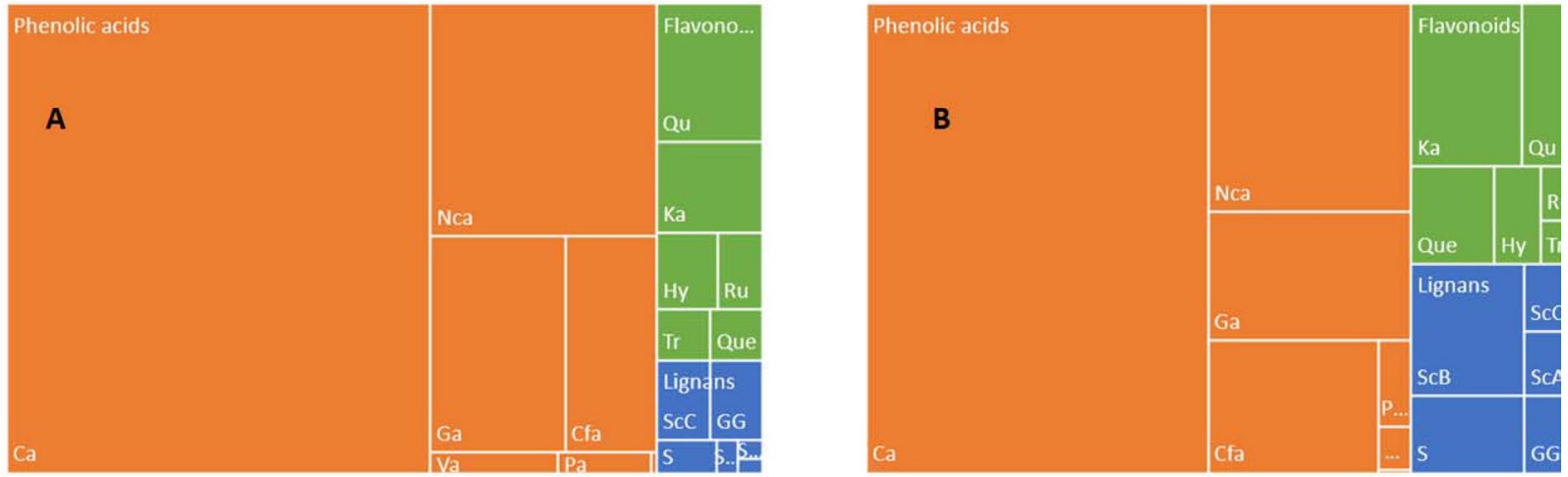

- Lignans " Phenolic acids $=$ Flavonoids

(c)
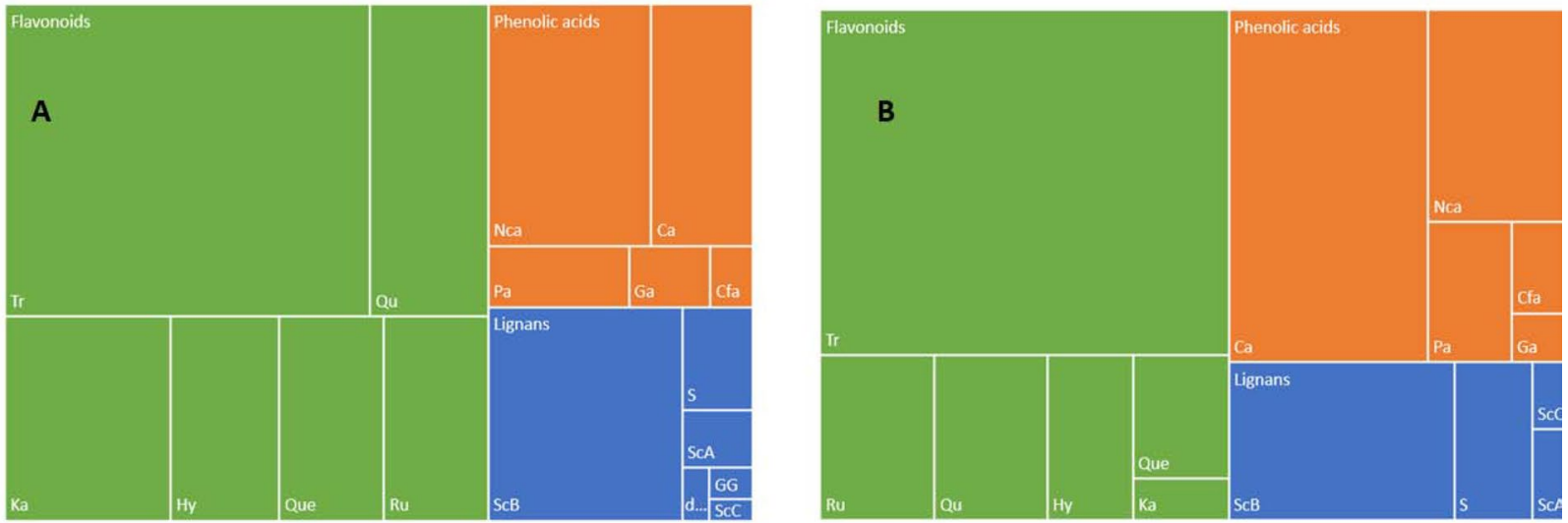

- Lignans $=$ Phenolic acids $=$ Flavonoids

Fig. 3 A treemap chart of: lignans ( $S$ Schisandrin, $G G$ Gomisin G, $S c A$ Schisantherin A, $S c B$ Schisantherin B, $d S$ deoxyschisandrin, $S c C$ Schisandrin C), phenolic acids ( $G a$ gallic acid, Nca neochlorogenic acid, $\mathrm{Ca}$ caftaric acid, $\mathrm{Pa}$ protocatechuic acid, $\mathrm{Cfa}$ caffeic acid), and flavonoids ( $H y$ hyperoside, $R u$ rutoside, $T r$ trifolin, $Q u$ quercitrin, Que quercetin, $K a$ kaempferol. Each rectangle represents amount in $\mathrm{mg} / 100 \mathrm{~g}$ DW. a In microshoot cultures, A-minimal, B-maximal content, see Table 1 for details; $\mathbf{b}$ in callus cultures, A-minimal, $\mathrm{B}$-maximal content, see Table 2 for details; c leaves harvested from parent plant in different vegetation periods, A-May, B-September, see Table 3 for details

from $0.46 \mathrm{mg} / 100 \mathrm{~g} \mathrm{DW}$ (10 days) to $17.49 \mathrm{mg} / 100 \mathrm{~g}$ DW (20 days), from $1.24 \mathrm{mg} / 100 \mathrm{~g} \mathrm{DW}$ (10 days) to $10.25 \mathrm{mg} / 100 \mathrm{~g}$ DW (20 days), and from $0.29 \mathrm{mg} / 100 \mathrm{DW}$ (10 days) to $7.29 \mathrm{mg} / 100 \mathrm{~g}$ DW (30 days), respectively (Tables 2 and S6, Fig. 3b). 
Table 1 Comparison of the contents $(\mathrm{mg} / 100 \mathrm{~g}$ DW $\pm \mathrm{SD})$ of dibenzocyclooctadiene lignans, phenolic acids and flavonoids in S. henryi microshoot cultures

\begin{tabular}{|c|c|c|c|c|c|}
\hline Compounds & $\begin{array}{l}\text { Minimal-maximal content }(\mathrm{mg} / 100 \mathrm{~g} \\
\mathrm{DW} \pm \mathrm{SD})\end{array}$ & $\begin{array}{l}\text { Fold incerase in } \\
\text { content }\end{array}$ & $\begin{array}{l}\text { Variant of MS } \\
\text { medium }^{\mathrm{a}}\end{array}$ & $\begin{array}{l}\text { Optimal growth } \\
\text { period (days) }\end{array}$ & Gi index $(\%)$ \\
\hline \multicolumn{6}{|l|}{ Lignans } \\
\hline Schisandrin & $6.53 \pm 0.06$ to $61.24 \pm 0.23^{*}$ & 9.37 & $\mathrm{~F}$ & 10 & 47.61 \\
\hline Gomisin G & $3.61 \pm 3.61$ to $18.20 \pm 0.18^{*}$ & 5.04 & $\mathrm{C}$ & 30 & 73.14 \\
\hline Schisantherin A & $31.50 \pm 0.25$ to $143.74 \pm 0.43^{*}$ & 4.56 & $\mathrm{~F}$ & 10 & 47.61 \\
\hline Schisantherin B & $140.21 \pm 0.36$ to $622.59 \pm 0.57 *$ & 4.44 & $\mathrm{~F}$ & 10 & 47.61 \\
\hline Deoxyschisandrin & $0.14 \pm 0.06$ to $1.03 \pm 0.18^{*}$ & 7.36 & A & 10 & 38.28 \\
\hline Schisandrin $\mathrm{C}$ & $3.27 \pm 0.07$ to $28.61 \pm 0.23^{*}$ & 8.75 & $\mathrm{~F}$ & 10 & 47.61 \\
\hline Total content & $213.78 \pm 1.58$ to $873.71 \pm 1.72 *$ & 4.09 & $\mathrm{~F}$ & 10 & 47.61 \\
\hline \multicolumn{6}{|l|}{ Phenolic acids } \\
\hline Gallic acid & $31.50 \pm 2.03$ to $59.86 \pm 2.42^{*}$ & 1.9 & B & 30 & 75.13 \\
\hline Neochlorogenic acid & $102.85 \pm 3.91$ to $472.82 \pm 6,47^{*}$ & 4.6 & B & 30 & 75.13 \\
\hline Caftaric acid & $68.59 \pm 2.98$ to $370.81 \pm 6.04^{*}$ & 5.41 & $\mathrm{~F}$ & 30 & 63.44 \\
\hline Chlorogenic acid & $0.44 \pm 0.04$ to $33.71 \pm 0.03^{*}$ & 76.61 & A & 10 & 38.27 \\
\hline Caffeic acid & $3.93 \pm 0.37$ to $30.02 \pm 0.28^{*}$ & 7.64 & A & 10 & 38.27 \\
\hline Vanillic acid & $0.17 \pm 0.01$ to $10.35 \pm 0.27^{*}$ & 60.88 & B & 10 & 48.45 \\
\hline Syringic acid & $3.73 \pm 0.27$ to $34.50 \pm 2.48^{*}$ & 9.25 & B & 30 & 75.13 \\
\hline Total content & $268.29 \pm 12.09$ to $840.89 \pm 16.91^{*}$ & 6.94 & $\mathrm{~B}$ & 30 & 75.13 \\
\hline \multicolumn{6}{|l|}{ Flavonoids } \\
\hline Hyperoside & $11.38 \pm 0.35$ to $47.56 \pm 2.08^{*}$ & 4.18 & $\mathrm{~B}$ & 10 & 48.45 \\
\hline Rutoside & $19.88 \pm 0.27$ to $88.83 \pm 3.28^{*}$ & 4.47 & B & 10 & 48.45 \\
\hline Trifolin & $15.77 \pm 0,30$ to $138.56 \pm 1.92^{*}$ & 8.79 & $\mathrm{~B}$ & 10 & 48.45 \\
\hline Quercitrin & $33.78 \pm 0.48$ to $122.54 \pm 2.58^{*}$ & 3.63 & B & 10 & 48.45 \\
\hline Quercetin & $2.35 \pm 0.02$ to $46.20 \pm 3.11^{*}$ & 19.66 & $\mathrm{~F}$ & 30 & 63.44 \\
\hline Kaempferol & $6.25 \pm 0.19$ to $26.06 \pm 0.44^{*}$ & 4.17 & $\mathrm{E}$ & 10 & 38.79 \\
\hline Total content & $100.10 \pm 2.62$ to $421.98 \pm 10.51^{*}$ & 4.21 & $\mathrm{~B}$ & 10 & 48.45 \\
\hline
\end{tabular}

The values marked with * differ statistically according to the Student's $\mathrm{t}$ test, $\alpha<0.05, \mathrm{n}=15$

${ }^{\mathrm{a}} \mathrm{A}-1 \mathrm{mg} / \mathrm{l} \mathrm{BA}$ and $1 \mathrm{mg} / \mathrm{lBA}, \mathrm{B}-1 \mathrm{mg} / \mathrm{l} \mathrm{BA}$ and $1 \mathrm{mg} / \mathrm{l} \mathrm{IBA}$ and $0.25 \mathrm{mg} / \mathrm{l} \mathrm{GA}, \mathrm{C}-0.25 \mathrm{mg} / \mathrm{l} \mathrm{BA}$ and $2 \mathrm{mg} / \mathrm{IBA}, \mathrm{D}-0.25 \mathrm{mg} / \mathrm{BA}$ and $3 \mathrm{mg} / \mathrm{l} \mathrm{IBA}, \mathrm{E}-2 \mathrm{mg} / \mathrm{l} \mathrm{BA}$ and $1 \mathrm{mg} / \mathrm{l} \mathrm{IBA}, \mathrm{F}-0.5 \mathrm{mg} / \mathrm{l} \mathrm{BA}$ and $2 \mathrm{mg} / \mathrm{l} \mathrm{IBA}$

The obtained total amounts of dibenzocyclooctadiene lignans depended on the duration of the growth cycle. The lowest total amount $(9.19 \mathrm{mg} / 100 \mathrm{~g} \mathrm{DW})$ was confirmed for callus biomass collected after 10 days of growth. The highest total amount of lignans (43.19 mg/100 g DW) was recorded for extracts from callus cultures harvested after 20 days of cultivation (Tables 2 and S6, Fig. S3a).

\section{Parent plant material}

The same 6 out of 9 tested dibenzocyclooctadiene lignans as in the in vitro cultures were present in extracts from the leaves of the $S$. henryi parent plant: schisandrin, gomisin $\mathrm{G}$, schisantherin A, schisantherin B, deoxyschisandrin, schisandrin $\mathrm{C}$ (Table 3, Fig. 3c). The quantitatively dominant individual compounds were: schisantherin B (48.99 mg/100 g DW, leaves harvested in May), schisandrin $(11.25 \mathrm{mg} / 100 \mathrm{~g}$ DW, leaves harvested in September) and schisantherin A $(4.75 \mathrm{mg} / 100 \mathrm{~g}$ DW, leaves harvested in May). The total amounts of lignans in the leaf extracts were $66.76 \mathrm{mg} / 100 \mathrm{~g}$ DW for the leaves harvested in May, and $56.83 \mathrm{mg} / 100 \mathrm{~g}$ DW for those harvested in September (Table 3, Fig. 3c).

\section{Production of phenolic acids}

\section{Microshoot cultures}

The effects of PGRs and duration of growth period on the yield of phenolic acid production were evident. In all extracts from microshoot cultures, 7 of the 24 tested compounds were detected-gallic, neochlorogenic, caftaric, chlorogenic, caffeic, syringic and vanillic acids (Tables 1 and S7-S9). The amounts of individual compounds varied, ranging from $0.19 \mathrm{mg} / 100 \mathrm{~g}$ DW (vanillic acid, MS variant E, 10 days) to $472.82 \mathrm{mg} / 100 \mathrm{~g}$ DW (neochlorogenic acid, MS variant B, 30 days) (Tables 1 and S7-S9, Fig. 3b). The quantitatively main compounds in all tested samples 
were: neochlorogenic, caftaric and gallic acids. Their maximal contents were: $472.82 \mathrm{mg} / 100 \mathrm{~g} \mathrm{DW}$ (30 days, variant $\mathrm{B}), 370.81 \mathrm{mg} / 100 \mathrm{~g} \mathrm{DW}$ (30 days, variant $\mathrm{F}$ ), and $59.86 \mathrm{mg} / 100 \mathrm{~g} \mathrm{DW}$ (30 days, variant B), respectively (Tables 1 and S7-S9).

The obtained total amounts of phenolic acids clearly depended on the MS medium variant and the duration of growth period. The lowest total amount $(268.29 \mathrm{mg} / 100 \mathrm{~g}$ DW) was obtained in extracts from the biomass collected after 20 days of growth on variant $\mathrm{C}$ (Tables 1 and S7-S9, Fig. S2b). The highest total amount of phenolic acids $(840.89 \mathrm{mg} / 100 \mathrm{~g} \mathrm{DW})$ was recorded in extracts from microshoots from MS medium variant $\mathrm{B}$, harvested after 30 days of cultivation (Tables 1 and S7-S9, Fig. S2b).

\section{Callus cultures}

In all extracts from callus cultures the same phenolic acids as in the microshoot cultures were detected (Tables 2 and $\mathrm{S} 10)$. The amounts of individual compounds varied with the duration of the growth period, ranging from $0.07 \mathrm{mg} / 100 \mathrm{~g}$ DW (syringic acid, 10 days) to $187.91 \mathrm{mg} / 100 \mathrm{~g} \mathrm{DW}$ (caftaric acid, 10 days). The quantitatively main compounds in all tested samples were: caftaric, neochlorogenic and gallic acids. Their maximal amounts were: $187.91 \mathrm{mg} / 100 \mathrm{~g}$ DW (10 days), $49.52 \mathrm{mg} / 100 \mathrm{~g}$ DW (10 days), and $30.58 \mathrm{mg} / 100 \mathrm{~g}$ DW (10 days), respectively (Tables 2 and S10, Fig. 3b).

The obtained total amounts of phenolic acids depended on the duration of cultivation. The lowest total amount $(192.25 \mathrm{mg} / 100 \mathrm{~g} \mathrm{DW})$ was obtained in callus biomass collected after 20 days of growth. The highest total amount of phenolic acids (296.66 mg/100 g DW) was recorded in extracts from callus cultures harvested after 10 days of cultivation (Tables 2 and S10, Fig. S3b).

\section{Parent plant material}

The presence of 5 out of the 24 tested compounds was confirmed in extracts of leaves of the $S$. henryi parent plant: gallic, neochlorogenic, caftaric, protocatechuic and caffeic acids (Table 3, Fig. 3c). The quantitatively main individual compounds were: caftaric acid $(64.16 \mathrm{mg} / 100 \mathrm{~g}$ DW, leaves harvested in September), neochlorogenic acid (46.64 mg/100 g DW, leaves harvested in May) and protocatechuic acid $(10.79 \mathrm{mg} / 100 \mathrm{~g} \mathrm{DW}$, leaves harvested in September). The total amounts of phenolic acids in the leaf extracts were $94.70 \mathrm{mg} / 100 \mathrm{~g} \mathrm{DW}$ for the material harvested in May, and $127.20 \mathrm{mg} / 100 \mathrm{~g} \mathrm{DW}$ for the leaves harvested in September (Table 3, Fig. 3c).

\section{Production of flavonoids}

\section{Microshoot cultures}

The concentrations of PGRs in the tested MS media variants and the duration of the growth period affected the flavonoid content in agar microshoot cultures. In all cultures, 6 of the 14 tested compounds were detected (mainly quercetin and kaempferol derivatives)-3-quercetin galactoside (hyperoside), 3-quercetin glucoramnoside (rutoside), kaempferol3-galactoside (trifolin), quercetin-3-rhamnoside (quercitrin), and quercetin and kaempferol (Tables 1 and S11-S13). The amounts of individual compounds depended on the medium variant and duration of the growth cycle, ranging from $2.35 \mathrm{mg} / 100 \mathrm{~g}$ DW (quercetin, MS variant F, 20 days) to $138.56 \mathrm{mg} / 100 \mathrm{~g} \mathrm{DW}$ (trifolin, MS variant $\mathrm{B}, 10$ days). The quantitatively dominant compounds in all samples were: trifolin (138.56 mg/100 g DW, 10 days, variant B), quercitrin $(122.54 \mathrm{mg} / 100 \mathrm{~g} \mathrm{DW}, 10$ days, variant $\mathrm{B})$ and rutoside ( $88.83 \mathrm{mg} / 100 \mathrm{~g} \mathrm{DW}, 10$ days, variant B), respectively (Tables 1 and S11-S13, Fig. 3a).

The obtained total amounts of flavonoids depended on the medium variant and the applied duration of growth cycles. The lowest total amount ( $110.10 \mathrm{mg} / 100 \mathrm{~g} \mathrm{DW})$ was documented in extracts of the biomass cultured for 20 days on variant D of MS medium. The highest total amount of flavonoids $(421.98 \mathrm{mg} / 100 \mathrm{~g} \mathrm{DW})$ was confirmed in extracts from microshoots cultivated on MS medium variant $\mathrm{B}$, harvested after 10 days of cultivation (Tables 1 and S11-S13, Fig. S2c).

\section{Callus cultures}

In all extracts from callus cultures, 6 of the 14 tested flavonoids were detected. They were the same ones as in microshoot cultures (Tables 2 and S14). The amounts of individual main compounds depended on the duration of the growth cycles, ranging from $1.64 \mathrm{mg} / 100 \mathrm{~g} \mathrm{DW}$ (quercetin, 20 days) to $21.16 \mathrm{mg} / 100 \mathrm{~g}$ DW (kaempferol, 30 days). The quantitatively main compounds in all samples were: kaempferol, quercitrin and quercetin. Their maximal amounts were: $21.16 \mathrm{mg} / 100 \mathrm{~g}$ DW (30 days), $18.87 \mathrm{mg} / 100 \mathrm{~g} \mathrm{DW}$ (10 days), and $9.55 \mathrm{mg} / 100 \mathrm{~g} \mathrm{DW}$ (10 days), respectively (Tables 2 and S14, Fig. 3b).

The obtained total amounts of flavonoids depended on the duration of culture growth. The lowest total amount (23.57 mg/100 g DW) was documented in extracts of the biomass cultured for 20 days. The highest total flavonoid content $(59.03 \mathrm{mg} / 100 \mathrm{~g} \mathrm{DW})$ was confirmed in extracts from callus cultures harvested after 30 days of cultivation (Tables 2 and S14, Fig. S3c). 
Table 2 Comparison of the contents $(\mathrm{mg} / 100 \mathrm{~g} \mathrm{DW} \pm \mathrm{SD})$ of dibenzocyclooctadiene lignans, phenolic acids and flavonoids in $S$. henryi callus cultures

\begin{tabular}{|c|c|c|c|c|}
\hline Compounds & $\begin{array}{l}\text { Minimal and maximal content } \\
(\mathrm{mg} / 100 \mathrm{~g} \mathrm{DW} \pm \mathrm{SD})\end{array}$ & $\begin{array}{l}\text { Fold } \\
\text { increase in } \\
\text { content }\end{array}$ & $\begin{array}{l}\text { Optimal } \\
\text { growth period } \\
\text { (days) }\end{array}$ & Gi index $(\%)$ \\
\hline \multicolumn{5}{|l|}{ Lignans } \\
\hline Schisandrin & $1.24 \pm 0.02$ to $10.25 \pm 2.06^{*}$ & 8.27 & 20 & 87.59 \\
\hline Gomisin $\mathrm{G}$ & $2.58 \pm 0.05$ to $5.11 \pm 0.14^{*}$ & 1.98 & 30 & 91.24 \\
\hline Schisantherin A & $0.29 \pm 0.36$ to $7.29 \pm 0.47^{*}$ & 25.14 & 30 & 91.34 \\
\hline Schisantherin B & $0.46 \pm 0.26$ to $17.49 \pm 7.78^{*}$ & 38.02 & 20 & 87.59 \\
\hline Deoxyschisandrin & $0.20 \pm 0.02$ to $3.63 \pm 0.27 *$ & 18.15 & 20 & 87.59 \\
\hline Schisandrin C & $2.69 \pm 0.74$ to $7.71 \pm 2.36^{*}$ & 2.87 & 20 & 87.59 \\
\hline Total content & $9.19 \pm 2.18$ to $43.18 \pm 14.02^{*}$ & 4.70 & 20 & 87.59 \\
\hline \multicolumn{5}{|l|}{ Phenolic acids } \\
\hline Gallic acid & $18.44 \pm 1.43$ to $30.58 \pm 3.06^{*}$ & 1.66 & 10 & 82.55 \\
\hline Neochlorogenic acid & $33.14 \pm 0.34$ to $49.52 \pm 1.14 *$ & 1.49 & 10 & 82.55 \\
\hline Caftaric acid & $125.27 \pm 7.19$ to $187.91 \pm 7.94^{*}$ & 1.50 & 10 & 82.55 \\
\hline Chlorogenic acid & $1.17 \pm 0.06$ to $3.33 \pm 2.88^{*}$ & 2.85 & 10 & 82.55 \\
\hline Caffeic acid & $12.50 \pm 0.21$ to $26.34 \pm 5.99^{*}$ & 2.11 & 30 & 91.34 \\
\hline Vanillic acid & $1.61 \pm 0.01$ to $1.68 \pm 0.08^{*}$ & 1.04 & 30 & 91.34 \\
\hline Syringic acid & $0.07 \pm 0.01$ to $0.10 \pm 0.02 *$ & 1.43 & 30 & 91.34 \\
\hline Total content & $192.25 \pm 9.37$ to $296.66 \pm 15.18^{*}$ & 1.54 & 10 & 82.55 \\
\hline \multicolumn{5}{|l|}{ Flavonoids } \\
\hline Hyperoside & $2.93 \pm 0.04$ to $5.23 \pm 0.24^{*}$ & 1.78 & 10 & 82.55 \\
\hline Rutoside & $2.15 \pm 0.24$ to $5.20 \pm 0.16^{*}$ & 2.42 & 30 & 91.34 \\
\hline Trifolin & $1.72 \pm 0.12$ to $4.07 \pm 0.11 *$ & 2.37 & 10 & 82.55 \\
\hline Quercitrin & $9.17 \pm 0.32$ to $18.87 \pm 1.19^{*}$ & 11.51 & 10 & 82.55 \\
\hline Quercetin & $1.64 \pm 0.28$ to $9.55 \pm 0.17 *$ & 5.82 & 10 & 82.55 \\
\hline Kaempferol & $5.97 \pm 0.34$ to $21.16 \pm 3.22^{*}$ & 3.54 & 30 & 91.34 \\
\hline Total content & $23.57 \pm 1.33$ to $59.03 \pm 6.09^{*}$ & 2.50 & 30 & 91.34 \\
\hline
\end{tabular}

The values marked with * differ statistically according to the Student's $\mathrm{t}$ test, $\alpha<0.05, \mathrm{n}=10$

\section{Parent plant material}

The same 6 of the 14 tested flavonoid compounds as in the in vitro cultures were confirmed in the extracts of leaves (harvested in May and September) of the S. henryi parent plant (Table 3, Fig. 3c). The quantitatively main individual compounds were: trifolin $(134.58 \mathrm{mg} / 100 \mathrm{~g} \mathrm{DW}$, leaves harvested in May), quercitrin (43.39 mg/100 g DW, harvested in May) and kaempferol $(40.05 \mathrm{mg} / 100 \mathrm{~g} \mathrm{DW}$, harvested in May). The total amounts of flavonoids in the leaf extracts were $294.69 \mathrm{mg} / 100 \mathrm{~g}$ DW for the leaves harvested in May, and $190.08 \mathrm{mg} / 100 \mathrm{~g}$ DW for the material harvested in September (Table 3, Fig. 3c).

\section{Discussion}

In the current study, the amounts of three groups of secondary metabolites: lignans, phenolic acids and flavonoids, in $S$. henryi biomass cultured in vitro were evaluated for the first time. Moreover, estimations were also performed, for comparative purposes, with the parent plant material (leaves collected at 2 different times of plant vegetation). Given the wide scope of experiments, which included a comprehensive optimization of combinations and concentrations of PGRs in the media, duration of growth period and type of cultures (microshoot and callus cultures), this work has a high biotechnological importance.

A significant impact of the applied conditions on biomass increases was proved for $S$. henryi in both microshoot and callus cultures. For microshoot cultures, the increase in biomass growth evaluated by the growth index $(\mathrm{Gi})$ ranged from $38.28 \%$ - for variant $\mathrm{A}$, with biomass harvested after 10 days, up to $75.58 \%$ - also for variant $\mathrm{A}$, and a 30 -day growth cycle (Fig. 2). These parameters were selected as the optimal "growth promoting" conditions. The maximum values of Gi for $S$. henryi microshoot cultures were quite high, higher than for in vitro cultures of e.g. Dionaea muscipula (Hook 2001), Thevetia peruviana (Zabala et al. 2010), or Genista tinctoria (Luczkiewicz et al. 2014) where the Gi values also were dependent on applied PGRs composition in the media. The maximum Gi value for $S$. chinensis agar 
Table 3 The amounts $(\mathrm{mg} / 100 \mathrm{~g} \mathrm{DW} \pm \mathrm{SD})$ of dibenzocyclooctadiene lignans, phenolic acids and flavonoids in $S$. henryi leaves harvested in different parent plant vegetation periods

\begin{tabular}{|c|c|c|}
\hline \multirow[t]{2}{*}{ Compounds } & \multicolumn{2}{|l|}{ Leaves } \\
\hline & May & September \\
\hline \multicolumn{3}{|l|}{ Lignans } \\
\hline Schisandrin & $8.62 \pm 0.95$ & $11.25 \pm 0.70^{*}$ \\
\hline Gomisin G & $1.62 \pm 0.51$ & $2.06 \pm 0.94$ \\
\hline Schisantherin A & $4.75 \pm 0.54$ & $4.45 \pm 0.52$ \\
\hline Schisantherin B & $48.99 \pm 4.73$ & $32.45 \pm 3.61 *$ \\
\hline Deoxyschisandrin & $1.70 \pm 0.55$ & $1.00 \pm 0.06$ \\
\hline Schisandrin C & $1.06 \pm 0.38$ & $5.62 \pm 0.63 *$ \\
\hline Total content & $66.74 \pm 7.66$ & $56.83 \pm 6.46$ \\
\hline \multicolumn{3}{|l|}{ Phenolic acids } \\
\hline Gallic acid & $5.91 \pm 0.41$ & $5.00 \pm 0.28$ \\
\hline Neochlorogenic acid & $46.64 \pm 1.69$ & $37.87 \pm 0.55^{*}$ \\
\hline Caftaric acid & $28.87 \pm 0.01$ & $64.16 \pm 0.61 *$ \\
\hline Protocatechuic acid & $10.23 \pm 0.12$ & $10.79 \pm 0.18$ \\
\hline Caffeic acid & $3.05 \pm 0.03$ & $9.38 \pm 0.03 *$ \\
\hline Total content & $94.70 \pm 2.26$ & $127.20 \pm 1.65^{*}$ \\
\hline \multicolumn{3}{|l|}{ Flavonoids } \\
\hline Hyperoside & $26.29 \pm 0.07$ & $12.85 \pm 0.52 *$ \\
\hline Rutoside & $25.15 \pm 0.21$ & $17.17 \pm 0.42 *$ \\
\hline Trifolin & $134.58 \pm 2.6$ & $128.87 \pm 4.6$ \\
\hline Quercitrin & $43.39 \pm 0.33$ & $16.86 \pm 0.51 *$ \\
\hline Quercetin & $25.23 \pm 0.08$ & $10.71 \pm 0.31 *$ \\
\hline Kaempferol & $40.05 \pm 0.57$ & $3.62 \pm 0.04 *$ \\
\hline Total content & $294.69 \pm 3.86$ & $190.08 \pm 6.4^{*}$ \\
\hline
\end{tabular}

The values marked with * differ statistically according to the Student's t test, $\alpha<0.05, \mathrm{n}=10$

microshoot cultures, obtained after optimization, was a little lower, about 65\% (Szopa and Ekiert 2013; Szopa et al. 2016, 2020), and also similarly for S. chinensis cv. Sadova-70\% (Szopa et al. 2018b, 2020).

For $S$. henryi callus cultures, the significant impact of the duration of growth period on biomass increase was confirmed, with Gi values ranging from 82.55 to $91.34 \%$, for callus harvested after 10 days and 30 days, respectively. The maximum Gi factor for $S$. chinensis callus cultures grown over 30 days was lower, equal to $82 \%$ (Szopa and Ekiert 2011, 2013).

The results of the phytochemical analyses (HPLC-DAD and UHPLC-MS/MS) confirmed the identical qualitative profile of lignans in all analyzed microshoot and callus cultures, and also in the leaves of the parent plant (Tables 1 and S2-S6).

The literature (Opletal et al. 2004; Zhu et al. 2007; Lu and Chen 2009) claimed that the HPLC analysis of schisandra lignans is often very difficult since it involves working with multi-ingredient extracts characterized by the presence of isomeric compounds with similar spectral properties. Nevertheless, the use of UHPLC-MS/MS techniques allowed us to accurately assess the lignan composition of $S$. henryi, including for the first time an analysis of the lignan profile in the biomass from $S$. henryi in vitro cultures, which indicated that they are able to produce compounds typical for the parent plant (Tables 1 and S2). Of the dibenzocyclooctadiene lignans, the following compounds were identified: schisantherin A and schisantherin B, angelogomisin Q, benzylogomisin $\mathrm{Q}$, gomisin $\mathrm{F}$, angelogomisin $\mathrm{O}$ and schisantherin D (Table S2). These structures had been confirmed before in $S$. henryi stem extracts, besides deoxyschisandrin, epischisandron, gomisin G, and isoanwulignan (Liu et al. 1988; Chen et al. 2005; Iu et al. 2009).

What is important is that with our UHPLC-MS/MS analyses, we also identified other compounds from two different groups of lignans, i.e. hernicine B from dibenzylbutane lignans and, from aryltetralin lignans, wulignan A1, wulignan A2, epiwulignan A1, enshicin, epienshicin and dimethylwulignan A1. Ganschisandrin, and hernicine A and B had been estimated before, but only in $S$. henryi stem extracts, and identified as unique to this species (Iu et al. 2009). The presence of hernicine B was confirmed by us, for the first time in the leaf and also in extracts from in vitro cultures. From the group of aryltetralin lignans, Liu et al. (1988) had identified wulignan A1, wulignan A2, dimethylwulignan A1 and epiwulignan A in stems, while enshicine and epienshicine were found in S. henryi shoots (Liu et al. 1984, 1988; Chen et al. 2003a). We also found them all and, for the first time, also in extracts from in vitro-grown biomass.

Moreover, with the UHPLC-MS/MS analyses we detected the triterpendoids: kadsuric acid and schisanhenric acid (Table S2). These metabolites had been found before in $S$. henryi leaves, shoots and fruits, besides nigranoic acid and isoschisanhenric acid (Lian-Niang and Hong 1986; Chen et al. 2003b, 2005, 2010). Although other triterpenoid compounds had also been identified in the leaves and shoots of S. henryi, such as henrischinins A, B and C, and henridilactones A, B, C and D (Chen et al. 2003a, 2010; Li et al. 2004), they were not found in the extracts from parent plant material as well as from in vitro cultures analyzed by us.

For S. henryi microshoot cultures, a significant influence of the applied conditions was proved on the individual and total yields of the detected lignans (Tables 1 and S3-S5, Figs. 3a and S2a). Noteworthy are the very high amounts of schisantherin B and schisantherin A in extracts from microshoots grown on variant medium $\mathrm{F}$ over 10-day growth periods (Tables 1 and S3, Fig. 3a). The total lignan content varied fourfold (from 213.78 to $873.71 \mathrm{mg} / 100 \mathrm{~g} \mathrm{DW}$ ) depending on the applied conditions (Table 1, Fig. S2a). The maximum total amount of lignans was found for microshoots cultured on medium variant $\mathrm{F}$ (containing $0.5 \mathrm{mg} / \mathrm{l} \mathrm{BA}$ and $2 \mathrm{mg} / \mathrm{lBA}$ ) over a 10-day growth period. 
The qualitative and quantitative composition of PGRs in the media, as well as duration of growth periods, have been proven to influence secondary metabolite production in vitro (Kozai et al. 1997; Hussain et al. 2012). NAA significantly increased shoot proliferation and influenced tannins, flavonoids and phenols accumulation in Huernia hystrix in vitro cultures (Amoo and van Staden 2013). In Aconitum violaceum shoot culture differences in cytokinin (BA, Kin, TDZ) concentrations, and longer durations of growth periods (8 weeks) significantly affected aconitine production (Janhvi et al. 2013). In Stevia rebaudiana tissue the increase of total phenols and flavonoids production varied with PGRs and BA either with $\mathrm{GA}_{3}$ or IAA gave best results (Radić et al. 2016). In Linum usitatissimum callus cultures NAA, BA and TDZ influenced production of specific lignans: secoisolariciresinol diglucoside, matairesinol, secoisolariciresinol, lariciresinol diglucoside, and the total phenolic content (Anjum et al. 2017).

The biomass from $S$. henryi callus cultures was found to contain the same lignans as in microshoot cultures, but their amounts were many times lower than in cultures with a higher degree of organogenesis (Table 2). The highest total lignan content was found for callus culture biomass harvested after 20 days of cultivation. The maximum total amount of lignans detected in the $S$. henryi microshoot cultures was more than 20 times higher than for callus cultures. For callus cultures, the effect of culture duration on the amounts of individual lignans and their total content in the biomass was evident, varying from 1.89 to $38.02 \mathrm{mg} / 100 \mathrm{~g}$ DW, and from 9.19 to $43.18 \mathrm{mg} / 100 \mathrm{~g} \mathrm{DW}$, respectively (Table S4). Noteworthy are the significant amounts of schisantherin B (17.49 mg/100 g DW) and schisandrin $(10.25 \mathrm{mg} / 100 \mathrm{~g} \mathrm{DW})$ in extracts from biomass harvested after 20 days, and of schisantherin $\mathrm{A}$ in those harvested after 30 days of culture $(7.29 \mathrm{mg} / 100 \mathrm{~g} \mathrm{DW})$ compared to the other lignans, whose amounts in $100 \mathrm{~g}$ DW did not exceed $3 \mathrm{mg}$ (Tables 2 and S6, Figs. $3 \mathrm{~b}$ and S3a).

The influence of degree of organogenesis on secondary metabolite production is one of the main factors to be tested to optimize in vitro cultures (Charlwood et al. 1990; Karuppusamy 2009; Hussain et al. 2012). Usually, highly differentiating cultures (microshoots, roots) produce higher amounts of metabolites, as reported for Aronia melanocarpa, where the highest amounts of phenolic acids were found in shoot cultures (Szopa et al. 2013), and in shoot cultures of Bacopa monniera where the bacoside A content was 4 times higher than in callus cultures (Parale et al. 2010). A higher content of flavonoids, as well as volatile constituents in essential oil was obtained for Lallemantia iberica shoot cultures, while the callus of this plant was efficient for total phenol production (Pourebad et al. 2015). Likewise here, only the production of phenolic acids was higher for $S$. henryi callus cultures (Table 2, Fig. 3b).
No information was found in the literature on the accumulation of lignans in $S$. henryi in vitro cultures. Comparing the results of qualitative and quantitative analyses obtained for in vitro cultures of $S$. henryi with those for the best-known species from the Schisandraceae family-S. chinensis, and its cultivar-S. chinensis cv. Sadova, both qualitative and quantitative differences were found. Particularly noteworthy are the extremely high amounts of schisantherin B and A in microshoot cultures of $S$. henryi. The schisantherin B content $(622.59 \mathrm{mg} / 100 \mathrm{~g} \mathrm{DW})$ was about 110 -fold higher than in extracts from biomass of $S$. chinensis microshoot cultures (max. $5.70 \mathrm{mg} / 100 \mathrm{~g} \mathrm{DW}$ ) (Szopa et al. 2016, 2020) and 17-fold higher than in extracts from biomass of S. chinensis cv. Sadova cultures (max. $35.64 \mathrm{mg} / 100 \mathrm{~g} \mathrm{DW}$ ) (Szopa et al. 2018b). For schisantherin A, its maximum amount in S. henryi microshoot cultures $(143.74 \mathrm{mg} / 100 \mathrm{~g} \mathrm{DW})$ was about 50-fold higher than for $S$. chinensis microshoot cultures (max. $2.97 \mathrm{mg} / 100 \mathrm{~g} \mathrm{DW}$ ) (Szopa et al. 2016, 2020) and about 20 -fold higher than for in vitro cultures of $S$. chinensis cv. Sadova (max. $7.99 \mathrm{mg} / 100 \mathrm{~g}$ DW) (Szopa et al. 2018b).

In our research, the highest amount of an individual lignan in callus cultures of $S$. henryi was also confirmed for schisantherin B (17.49 mg/100 g DW). For S. chinensis callus cultures this compound had not been previously analyzed by other teams (Březinová et al. 2010; Kohda et al. 2012; Szopa et al. 2020). Another dominant lignan in S. henryi callus cultures was schisandrin, whose amount reached max. $10.25 \mathrm{mg} / 100 \mathrm{~g}$ DW. Conflictingly, compared with callus cultures of $S$. chinensis tested earlier, this content was about 11 times higher $(0.90 \mathrm{mg} / 100 \mathrm{~g} \mathrm{DW})$ than found by Březinová et al. (2010) but about 7 times lower than measured (70.54 mg/100 g DW) by Szopa and Ekiert (2015).

A comparative analysis of the lignan content in the leaves of the parent plant harvested in May and September was carried versus results from the optimized $S$. henryi in vitro cultures, to assess their usefulness as an alternative source of biologically active lignans and phenolic compounds.

The composition of dibenzocyclooctadiene lignans from $S$. henryi in vitro cultures was the same as in the leaves of the parent plant. The maximum total amount of lignans obtained in microshoot extracts was about 13 -fold higher than in extracts from the leaves collected in May, and about 15-fold higher than in extracts from leaves collected in September (Tables 1, 3, Fig. 3c). The obtained maximum amounts of the main dibenzocyclooctadiene lignans: schisandrin, gomi$\sin \mathrm{G}$ and schisantherin $\mathrm{B}$, were respectively about 5, 9 and 13 times higher in biomass extracts from microshoot cultures than in the leaves of the parent plant.

Comparing the results of analyses of dibenzocyclooctadiene lignans in leaf extracts of $S$. henryi with those for the $S$. chinensis species and its cultivar-S. chinensis cv. Sadova, differences were found in the qualitative composition. S. chinensis and its cultivar are more abundant in 
dibenzocyclooctadiene lignans, the most characteristic of which were identified as "Schisandra chinensis lignans", such as: schisandrin (the most abundant), gomisin A, gomisin G, schisantherin A, schisantherin B, schisanhenol, deoxyschisandrin, $\gamma$-schisandrin and schisandrin C (Szopa et al. 2016, 2018b). Those identified here in $S$. henryi leaves were: schisandrin, gomisin G, schisantherin A, schisantherin B, deoxyschisandrin and schisandrin $\mathrm{C}$. Their amounts were many times lower than in extracts of $S$. chinensis and $S$. chinensis cv. Sadova. However, it is worth noting the high amount of schisantherin B in extracts from $S$. henryi leaves, about 14 times higher than in leaf extracts of $S$. chinensis and S. chinensis cv. Sadova (Szopa et al. 2016, 2018b).

In our study, we examined the phenolic profile of extracts from $S$. henryi in vitro cultures aimed at qualitative and quantitative analyses of phenolic acids and flavonoids using the HPLC-DAD method. Depending on the medium and duration of the growth cycle, the amounts of individual phenolic acids and their total content in microshoot biomass extracts varied from 0.17 to $472.82 \mathrm{mg} / 100 \mathrm{~g} \mathrm{DW}$, and from 268.29 to $840.89 \mathrm{mg} / 100 \mathrm{~g}$ DW, respectively (Tables 2 and S7-S9). Noteworthy are the very high amounts of neochlorogenic acid in extracts from microshoots grown on medium variant $\mathrm{B}$, collected after 30 days, and of caftaric acid in extracts from microshoots grown on medium variant $\mathrm{F}$, harvested after 30 days. The highest total amount of phenolic acids was found in microshoot extracts grown on variant $B$ of the MS medium, harvested after a 30-day growth period (Table 2, Figs. 3a and S2b).

The examination of phenolic acids in callus cultures of $S$. henryi revealed the same phenolic acids composition as in microshoot cultures; however, their contents were many times lower than in organogenic cultures. The amounts of individual phenolic acids and their total contents in biomass extracts varied depending on the duration of the growth cycle (Tables 2 and S10, Figs. 3b and S3b). The highest total phenolic acid content was found in the callus extracts harvested after 10 days of culture. The maximum total amount of phenolic acids detected in the $S$. henryi microshoot cultures was 2.83 times higher than for callus cultures. Noteworthy are the high amounts of caftaric acid in extracts from callus cultures harvested after 10 days of growth period, and neochlorogenic acid in extracts from biomass collected after a 10-day growth period (Tables 2 and S10).

Comparing the results of the phenolic acid analysis obtained here in extracts from $S$. henryi microshoot cultures with those from in vitro microshoot cultures of the pharmacopoeial S. chinensis and its cultivar S. chinensis cv. Sadova, both qualitative and quantitative differences were found. In S. chinensis microshoot extracts, seven phenolic acids had been detected, i.e. chlorogenic, gallic, p-hydroxybenzoic, protocatechuic, syringic, salicylic and vanillic acids (Szopa et al. 2019c). Particularly noteworthy are the high amounts of neochlorogenic and caftaric acids in extracts from S. henryi cultures. The neochlorogenic acid in $S$. chinensis cultures had not been analyzed previously (Mocan et al. 2016; Szopa et al. 2019c). In S. chinensis cv. Sadova agar microshoot cultures, the presence of eight phenolic acids had been confirmed-chlorogenic, cryptochlorogenic, gallic, neochlorogenic, protocatechuic, salicylic, syringic and vanillic acids (Szopa et al. 2019b). The amount of neochlorogenic acid (116.65 mg/100 g DW) in S. chinensis cv. Sadova was 4 times lower compared to $S$. henryi microshoot cultures. A high content was also obtained for caftaric acid in the extracts from $S$. henryi cultures, but this compound had not been detected before in in vitro cultures of $S$. chinensis and S. chinensis cv. Sadova (Mocan et al. 2016; Szopa et al. 2019b, c, 2020).

The comparative analyses of the amounts of phenolic acids in parent plant leaf extracts showed that their maximum total content for $S$. henryi microshoot extracts was almost ninefold higher than in extracts from leaves collected in May and almost sevenfold higher than in extracts from leaves collected in September. The maximum amounts of the main detected phenolic acids, i.e. neochlorogenic, caftaric and gallic, were respectively: 10, 6 and 10 times higher in in vitro cultures than in the leaves of the parent plant (Table 3, Fig. 3c). Neither vanillic nor syringic acid, which were found in vitro, were present in the leaves of the parent plant (Tables 1, 2, 3).

Comparing leaf extracts of $S$. henryi with those of $S$. chinensis (Szopa et al. 2019c) and S. chinensis cv. Sadova (Szopa et al. 2018b, 2019b), differences were apparent in the qualitative composition and in the amounts of phenolic acids. In S. chinensis and S. chinensis cv. Sadova leaf extracts, the dominant compound was chlorogenic acid, while it was not detected in extracts from $S$. henryi leaves, where the dominant compounds were neochlorogenic and caftaric acids.

The qualitative and quantitative analyses of polyphenolic compounds in $S$. chinensis leaf extracts performed by Mocan et al. (2014) had confirmed only the presence of one phenolic acid as in $S$. henryi-chlorogenic acidas the most abundant, while p-coumaric acid and ferulic acid, were detected too. Our earlier work on the composition of phenolic acids in S. chinensis leaves had revealed also the presence of chlorogenic acid, and of protocatechuic acid, salicylic acid and p-hydroxybenzoic acid (Szopa et al. 2019c, 2020). In the leaf extracts of $S$. chinensis cv. Sadova we had detected similar phenolic acids as in $S$. henryi, i.e., neochlorogenic acid, chlorogenic acid, vanillic acid, gallic acid and syringic acid. Moreover, cryptochlorogenic acid, salicylic acid and protocatechuic acid were estimated, too (Szopa et al. 2019b).

The main flavonoids in extracts from $S$. henryi in vitro cultures were quercetin and kaempferol derivatives: 3-quercetin galactoside (hyperoside), 3-quercetin 
glucoramnoside (rutoside), kaempferol-3-galactoside (trifolin), quercetin-3-rhamnoside (quercitrin), and quercetin and kaempferol (Tables 2 and S11-S14). The influence of PGRs compoition and of the duration of growth cycles on the individual and total amounts of flavonoids was evident, and their increases varied within wide ranges (Tables 2 and S11-S14, Figs. 3a and S3c). Worthy of note are the very high amounts of kaempferol-3-galactoside (trifolin) and quercetin-3-rhamnoside (quercitrin), and the maximum total amount of flavonoids, that were all detected in the extracts from microshoots grown on variant B of MS medium and harvested after a 10-day growth period (Table 2, Fig. 3a).

In extracts from $S$. henryi callus cultures, the amounts of flavonoids were many times lower than for the organogenic cultures. The main compounds were kaempferol and quercetin-3-rhamnoside (quercitrin). The highest total flavonoid content, found in extracts of callus biomass harvested after a 10-day growth period, was 7 times lower than for microshoot cultures (Fig. S3c).

We had tested $S$. chinensis microshoot cultures growing in different types of plant bioreactors for flavonoid content before (Szopa et al. 2019c), and we identified: kaempferol, quercetin-3-rhamnoside (quercitrin) and quercetin-3-O-rutinoside (rutoside). The highest total amount of flavonoids was found in microshoots maintained in a nutrient sprinkle bioreactor for 30 days. The highest content was confirmed for quercetin-3-rhamnoside (quercitrin), and this amount was 1.25 times lower than for the S. henryi microshoot cultures and 2.20 times higher than for the callus cultures.

We had also analyzed flavonoid accumulation in microshoot cultures of $S$. chinensis cv. Sadova, where we confirmed the presence of only quercetin-3-rhamnoside (quercitrin) and kaempferol (Szopa et al. 2018b). The maximum amount of quercitrin was 1.5 times lower than for $S$. henryi microshoot cultures, and 4.4 times higher than for callus cultures. The maximum amount of kaempferol in S. chinensis cv. Sadova microshoot cultures (Szopa et al. 2019b) was similar to its amounts in both the microshoot and callus cultures of S. henryi.

The analysis of flavonoid content in parent leaf extracts showed the same qualitative composition for in vitro and in vivo materials (Tables 1, 2, 3, Fig. 3c). The obtained maximum total flavonoid content in microshoot extracts was 1.4fold higher than in extracts of the leaves harvested in May and 2.2-fold higher than in extracts of the leaves harvested in September. The maximum amounts of the main flavonoids: trifolin and quercitrin, were respectively 1.02 and 2.82 times higher in biomass extracts from in vitro cultures than in the leaves of the parent plant.

The qualitative and quantitative analyses of polyphenolic compounds performed by Mocan et al. (2014) had confirmed in S. chinensis leaf extracts the presence of the flavonoid glycosides: isoquercitrin, quercitrin, rutoside and hyperoside, and of the flavonoid aglycones: kaempferol and quercetin. The estimation of flavonoid composition in S. chinensis leaves performed by us earlier had revealed differences in the qualitative composition; we had found the presence of two aglycones: myricetin and quercetin, and of two glycosides: quercetin-3-rhamnoside (quercitrin) and quercetin-3-Orutinoside (rutoside), and the main flavonoids were quercitrin and quercetin (Szopa et al. 2019c). The total flavonoid content in S. chinensis leaf extracts was comparable to that estimated in $S$. henryi leaves (Table 3, Fig. 3c). Comparing the results of flavonoid estimations in extracts from $S$. henryi leaves with the amounts of these compounds in the leaves of S. chinensis cv. Sadova (Szopa et al. 2019b), we found similar qualitative compositions and differences in the amounts of the tested flavonoids. The extracts from $S$. henryi leaves contained higher amounts of trifolin, which had not been detected in previous studies. On the other hand, the detected amounts of quercitrin and kaempferol were higher than in S. henryi leaves.

To conclude, the object of the study was the endemic plant species-S. henryi, of which initiation and optimization of in vitro cultures were performed for the first time. The different types of in vitro cultures (microshoot and callus), different durations of growth periods, and different concentrations of PGRs, were all proved to influence biomass growth and the production of secondary metabolites. In the methanolic extracts from biomass of the in vitro cultures, lignans, phenolic acids and flavonoids were detected. The highest total amount of lignans was obtained in the extracts from biomass of the microshoot cultures cultivated for 10 days on MS medium containing $0.5 \mathrm{mg} / \mathrm{l} \mathrm{BA}$ and $2 \mathrm{mg} / \mathrm{l}$ IBA. The main compounds were: schisantherin B and schisantherin. The highest total amount of phenolic acids was obtained in the extracts from biomass of the microshoot cultures cultivated for 30 days on MS medium with $1 \mathrm{mg} / \mathrm{l}$ BA, $1 \mathrm{mg} / \mathrm{l} \mathrm{IBA}$ and $0.25 \mathrm{mg} / \mathrm{GA}_{3}$. The main compounds were: neochlorogenic acid and caftaric acid. The highest total amount of flavonoids was obtained in extracts from biomass of the shoot cultures cultivated for 10 days on MS medium with $1 \mathrm{mg} / \mathrm{l} \mathrm{BA}, 1 \mathrm{mg} / \mathrm{lBA}$ and $0.25 \mathrm{mg} / \mathrm{l} \mathrm{GA}$. The main compounds were: kaempferol-3-galactoside (trifolin) and quercetin-3-rhamnoside (quercitrin).

In this work, the dibenzocyclooctadiene lignans profile was confirmed by the UHPLC-MS/MS method. Additionally, other types of lignans: dibenzylbutane lignans (hernicine B) and aryltetrahydrofuran lignans (wulignan A1, wulignan A2, epiwulignan A1, enshicine, epienshicine and dimethylwulignan A1) were estimated. The results of the UHPLC-MS/MS analyses also confirmed the presence of triterpenoids: kadsuric acid and schisanhenric acid.

The amounts of lignans, phenolic acids and flavonoids were determined in plant material grown in vivo (leaves of a male $S$. henryi specimen). The highest total amounts of these 
secondary metabolites in the methanolic leaf extracts were respectively 13,7 , and 1.4 times lower than in extracts from in vitro cultures. Obtaining a greater production of secondary metabolites in the biomass of in vitro cultures than in the parent plant is an excellent outcome of such experiments (Karuppusamy 2009; Murthy et al. 2014).

The meagre phytochemical papers and the lack of biotechnological works on $S$. henryi species make the subject of this work relevant and innovative. The prospect of further research will undoubtedly be further optimization of cultures, among others in the form of agitated cultures and then growing in plant bioreactors. Increasing the production of secondary metabolites could be obtained, inter alia, by the addition of exogenous precursors to the culture media or by elicitation experiments.

Author contributions AS, KJ, MB and MD carried out the experiments, compiled the data, performed the chemical and statistical analysis. AS, KJ, MD and HE corrected and revisited the manuscript. All the authors read and approved the manuscript in its final form.

Funding This study was funded by National Science Centre, Poland (Grant Number 2016/23/D/NZ7/01316).

\section{Compliance with ethical standards}

Conflict of interest All authors declares that they have no conflict of interest.

Ethical approval This article does not contain any studies with human participants or animals performed by any of the authors.

Open Access This article is licensed under a Creative Commons Attribution 4.0 International License, which permits use, sharing, adaptation, distribution and reproduction in any medium or format, as long as you give appropriate credit to the original author(s) and the source, provide a link to the Creative Commons licence, and indicate if changes were made. The images or other third party material in this article are included in the article's Creative Commons licence, unless indicated otherwise in a credit line to the material. If material is not included in the article's Creative Commons licence and your intended use is not permitted by statutory regulation or exceeds the permitted use, you will need to obtain permission directly from the copyright holder. To view a copy of this licence, visit http://creativecommons.org/licenses/by/4.0/.

\section{References}

Amoo SO, van Staden J (2013) Influence of plant growth regulators on shoot proliferation and secondary metabolite production in micropropagated Huernia hystrix. Plant Cell Tissue Organ Cult 112:249-256. https://doi.org/10.1007/s11240-012-0230-x

Anjum S, Abbasi BH, Hano C (2017) Trends in accumulation of pharmacologically important antioxidant-secondary metabolites in callus cultures of Linum usitatissimum L. Plant Cell Tissue Organ Cult 129:73-87. https://doi.org/10.1007/s11240-016-1158-3

Bhojwani SS, Razdan MK (1989) Plant tissue culture: theory and practice, vol 5, 1st edn. Elsevier, Delhi
Březinová L, Vlašínová H, Havel L et al (2010) Validated method for bioactive lignans in Schisandra chinensis in vitro cultures using a solid phase extraction and a monolithic column application. Biomed Chromatogr 24:954-960. https://doi.org/10.1002/ bmc. 1391

Charlwood B, Charlwood S, Molina-Tores J (1990) Accumulation of secondary compounds by organized plant cultures. In: Charlwood BV, Rhodes MJC (eds) Secondary products from plant tissue cultures. Clarendon Press, Oxford, pp 167-200

Chen Y-G, Wu Z-C, Lv Y-P et al (2003a) Triterpenoids from Schisandra henryi with cytotoxic effect on leukemia and Hela cells in vitro. Arch Pharm Res 26:912-916. https://doi.org/10.1007/ BF02980199

Chen Y-G, Wu Z-C, Lv Y-P et al (2003b) Triterpenoids from Schisandra henryi with cytotoxic effect on leukemia and hela cells in vitro. Arch Pharm Res 26:912-916. https://doi.org/10.1007/ BF02980199

Chen YG, Wu ZC, Gui SH et al (2005) Lignans from Schisandra henryi with DNA cleaving activity and cytotoxic effect on leukemia and Hela cells in vitro. Fitoterapia 76:370-373. https://doi. org/10.1016/j.fitote.2005.03.012

Chen YG, Zhang Y, Liu Y et al (2010) A new triterpenoid acid from Schisandra henryi. Chem Nat Compd 46:569-571. https://doi. org/10.1007/s10600-010-9677-3

Chen C-Y, Liu S-Y, Yan Y et al (2020) Candidate genes involved in the biosynthesis of lignan in Schisandra chinensis fruit based on transcriptome and metabolomes analysis. Chin J Nat Med. https ://doi.org/10.3724/SP.J.1009.2019.000000

Christophe W (2013) Lead compounds from medicinal plants for the treatment of cancer, 1st edn. Elsevier, London

Deng X, Chen X, Cheng W et al (2008) Simultaneous LC-MS quantification of 15 lignans in Schisandra chinensis (Turcz.) Baill. fruit. Chromatographia 67:559-566. https://doi.org/10.1365/ s10337-008-0589-3

European Directorate for the Quality of Medicines (2008) Schisandrae chinensis fructus. In: European Pharmacopoeia 6.0. Strasburg

European Directorate for the Quality of Medicines (2017) Schisandrae chinensis fructus. In: European Pharmacopoeia 9.0. Strasburg

Ellnain-Wojtaszek M, Zgórka G (1999) High-performance liquid chromatography and thin-layer chromatography of phenolic acids from Ginkgo biloba L. leaves collected within vegetative period. J Liq Chromatogr Relat Technol 22:1457-1471. https:// doi.org/10.1081/JLC-100101744

Hammond J, McGarvey P, Yusibov V (2000) Plant biotechnology: new products and applications. Springer, Berlin

Harborne JB (1984) Phytochemical methods. Springer, Dordrecht

He XG, Lian LZ, Lin LZ (1997) Analysis of lignan constituents from Schisandra chinensis by liquid chromatography-electrospray mass spectrometry. J Chromatogr A 757:81-87. https://doi.org/10.1016/ S0021-9673(96)00685-1

He X, Chen J, Mu Y et al (2020) The effects of inhibiting the activation of hepatic stellate cells by lignan components from the fruits of Schisandra chinensis and the mechanism of schisanhenol. J Nat Med 74:513-524. https://doi.org/10.1007/s11418-020-01394-w

Heleno SA, Martins A, Queiroz MJRP, Ferreira ICFR (2015) Bioactivity of phenolic acids: metabolites versus parent compounds: a review. Food Chem 173:501-513. https://doi.org/10.1016/j.foodc hem.2014.10.057

Hook ILI (2001) Naphthoquinone contents of in vitro cultured plants and cell suspensions of Dionaea muscipula and Drosera species. Plant Cell Tissue Organ Cult 67:281-285. https://doi. org/10.1023/A:1012708819212

Hussain MS, Fareed S, Ansari S et al (2012) Current approaches toward production of secondary plant metabolites. J Pharm Bioallied Sci 4:10-20. https://doi.org/10.4103/0975-7406.92725 
Ikeya Y, Taguchi H, Yosioka I, Kobayashi H (1979a) The constituents of Schizandra chinensis Baill. The cleavage of the methylenedioxy moiety with lead tetraacetate in benzene, and the structure of angeloylgomisin Q. Chem Pharm Bull 27:2536-2538

Ikeya Y, Taguchi H, Yosioka I, Kobayashi H (1979b) The constituents of Schizandra chinensis Baill. III. The structures of four new lignans, gomisin $\mathrm{H}$ and its derivative, angeloyl-, tigloyl- and benzoylgomisin H. Chem Pharm Bull 27:1576-1582

Ikeya Y, Taguchi H, Yosioka I (1980) The constituents of Schizandra chinensis Baill. VII. The structures of three new lignans, (-)-gomisin K1 and (+)-gomisins K2 and K3. Chem Pharm Bull 28:2422-2427

Ikeya Y, Ookawa N, Taguchi H, Yosioka I (1982a) The constituents of Schizandra chinensis Baill. XI. The structures of three new lignans, angeloylgomisin $\mathrm{O}$, and angeloyl- and benzoylisogomisin O. Chem Pharm Bull 30:3202-3206

Ikeya Y, Taguchi H, Yosioka I (1982b) The constituents of Schizandra chinensis Baill. XII. Isolation and structures of new lignans, gomisin $\mathrm{R}$, the absolute structures of wuweizisu $\mathrm{C}$ and isolation of schisantherin D. Chem Pharm Bull 30:3207-3211

Ikeya Y, Miki E, Okada M et al (1986) Benzoylgomisin Q and Benzylgomisin P, two new lignans from Schisandra sphenanthera Rehd. et Wils. Chem Pharm Bull 38:1408-1411

Ikeya Y, Taguchi H, Mitsuhashi H et al (1988) A lignan from Schizandra chinensis. Phytochemistry 27:569-573

Ikeya Y, Taguchi H, Yosioka I, Kobayashi H (1990) The structures of four new lignans, gomisin $\mathrm{N}$, gomisin $\mathrm{O}$, epigomisin $\mathrm{O}$ and gomisin $\mathrm{E}$, and transformation of gomisin $\mathrm{N}$ to deangeloylgomisin B. Chem Pharm Bull 38:1408-1411

Iu HL, Li-jia XU, Eng YP et al (2009) Two New Lignans from Schisandra henryi. Chem Pharm Bull 57:405-407. https://doi. org/10.1248/cpb.57.405

Janhvi MR, Balwant R, Anup C et al (2013) Influence of plant growth regulators on indirect shoot organogenesis and secondary metabolite production in Aconitum violaceum Jacq. African J Biotechnol 12:6287-6293. https://doi.org/10.5897/ajb2013.13390

Jian-Min Y, Yao-Zu C, Su-Ming H et al (1989) Ganschisandrine, a lignan from Schisandra sphenanthera. Phytochemistry 28:17741776. https://doi.org/10.1016/S0031-9422(00)97848-8

Jia-Sen L, Mei-Fen H, Yao-Liang G (1980) Studies on the constituents of Schisandra henryi Clarke. H. The structures of schisanhenrin and schisanhenric acid. Acta Chim Sin 38:363-370

Jia-Sen L, Yu-Ting M, Mei-Fen H (1988) Studies on the constituents of Schisandraceae plants in Shennongjia District. I. The constituents of Schisandra propinqua Hook. F. et Thoms, var. sinensis Oliv. Acta Chim Sin 46:345-348

Karuppusamy S (2009) A review on trends in production of secondary metabolites from higher plants by in vitro tissue, organ and cell cultures. J Med Plants Res 3:1222-1239

Kohda H, Ozaki M, Namera A (2012) Production of lignans in calluses of Schisandra chinensis. J Nat Med 66:373-376. https://doi. org/10.1007/s11418-011-0586-y

Kozai T, Kubota C, Jeong BR (1997) Environmental control for the large-scale production of plants through in vitro techniques. Plant Cell Tissue Organ Cult 51:49-56. https://doi.org/10.1023/A:10058 09518371

Li R, Shen Y, Xiang W, Sun H (2004) Four novel nortriterpenoids isolated from Schisandra henryi var. yunnanensis. Eur J Org Chem 2004:807-811. https://doi.org/10.1002/ejoc.200300560

Lian-Niang L, Hong X (1986) Henricine, a new tetrahydrofuran lignan from Schisandra henryi. Planta Med 52:493-494. https://doi. org/10.1055/s-2007-969265

Liu J-S, Tao Y, Huang M-F (1988) Studies on the constituents of Schisandra henryi V. The structures of wulignan A1,
A2, epiwulignan A1 and epischisandrone. J Chin Chem Soc $46: 483-488$

Liu G, Liu Y, Sun Y et al (2020) Lignans and terpenoids from the leaves of Schisandra chinensis. Chem Biodivers. https://doi. org/10.1002/cbdv.202000035

Lu Y, Chen DF (2009) Analysis of Schisandra chinensis and Schisandra sphenanthera. J Chromatogr A 1216:1980-1990. https://doi. org/10.1016/j.chroma.2008.09.070

Luczkiewicz M, Kokotkiewicz A, Glod D (2014) Plant growth regulators affect biosynthesis and accumulation profile of isoflavone phytoestrogens in high-productive in vitro cultures of Genista tinctoria. Plant Cell Tissue Organ Cult 118:419-429. https://doi. org/10.1007/s11240-014-0494-4

Mocan A, Crişan G, Vlase L et al (2014) Comparative studies on polyphenolic composition, antioxidant and antimicrobial activities of Schisandra chinensis leaves and fruits. Molecules 19:1516215179. https://doi.org/10.3390/molecules190915162

Mocan A, Schafberg M, Crisan G, Rohn S (2016) Determination of lignans and phenolic components of Schisandra chinensis (Turcz.) Baill. using HPLC-ESI-ToF-MS and HPLC-online TEAC: contribution of individual components to overall antioxidant activity and comparison with traditional antioxidant assays. J Funct Foods 24:579-594. https://doi.org/10.1016/j.jff.2016.05.007

Mohan J (2016) Protocols for in vitro cultures and secondary metabolite analysis of aromatic and medicinal plants, 2nd edn. Humana Press, New York

Murashige T, Skoog F (1962) A revised medium for rapid growth and bioassays with tobacco tissue cultures. Physiol Plant 15:473-497. https://doi.org/10.1016/S0031-9422(01)00179-0

Murthy HN, Lee E-J, Paek K-Y (2014) Production of secondary metabolites from cell and organ cultures: strategies and approaches for biomass improvement and metabolite accumulation. Plant Cell Tissue Organ Cult 118:1-16. https://doi.org/10.1007/s1124 0-014-0467-7

Nijveldt RJ, Nood E, Hoorn DE et al (2001) Flavonoids: a review of probable mechanisms of action and potential applications. Am J Clin Nutr 74:418-425. https://doi.org/10.1093/ajcn/74.4.418

Opletal L, Sovová H, Bártlová M (2004) Dibenzo[a, c]cyclooctadiene lignans of the genus Schisandra: importance, isolation and determination. J Chromatogr B 812:357-371. https://doi.org/10.1016/j. jchromb.2004.07.040

Parale A, Barmukh R, Nikam T (2010) Influence of organic supplements on production of shoot and callus biomass and accumulation of bacoside in Bacopa monniera (L.) Pennell. Physiol Mol Biol Plants 16:167-175. https://doi.org/10.1007/s1229 8-010-0018-6

Pourebad N, Motafakkerazad R, Kosari-Nasab M et al (2015) The influence of TDZ concentrations on in vitro growth and production of secondary metabolites by the shoot and callus culture of Lallemantia iberica. Plant Cell Tissue Organ Cult 122:331-339. https://doi.org/10.1007/s11240-015-0769-4

Radić S, Vujčić V, Glogoški M, Radić-Stojković M (2016) Influence of $\mathrm{pH}$ and plant growth regulators on secondary metabolite production and antioxidant activity of Stevia rebaudiana (Bert). Period Biol. https://doi.org/10.18054/PB.V118I1.3420

Saunders RMK (2000) Monograph of Schisandra (Schisandraceae). In: Saunders RMK (ed) Systematic botany monographs. American Society of Plant Taxonomists, Ann Arbor, pp 1-146

Sułkowska-Ziaja K, Maślanka A, Szewczyk A, Muszyńska B (2017) Physiologically active compounds in four species of Phellinus. Nat Prod Commun 12:363-336

Szopa A, Ekiert H (2011) Lignans in Schisandra chinensis in vitro cultures. Pharmazie 66:633-634

Szopa A, Ekiert H (2013) Production of deoxyschizandrin and $\gamma$-schizandrin in shoot-differentiating and undifferentiating callus cultures of Schisandra chinensis (Turcz.) Baill. 
(Chinese magnolia vine). J Biotechnol 165:209-213. https:// doi.org/10.1016/j.jbiotec.2013.03.010

Szopa A, Ekiert H (2015) Production of schisantherin A and gomisin $\mathrm{G}$ in in vitro cultures of Schisandra chinensis. Phytochem Lett 11:440-444. https://doi.org/10.1016/J.PHYTOL.2014.12.022

Szopa A, Ekiert H, Muszyńska B (2013) Accumulation of hydroxybenzoic acids and other biologically active phenolic acids in shoot and callus cultures of Aronia melanocarpa (Michx.) Elliott (black chokeberry). Plant Cell Tissue Organ Cult 113:323329. https://doi.org/10.1007/s11240-012-0272-0

Szopa A, Kokotkiewicz A, Marzec-Wróblewska U et al (2016) Accumulation of dibenzocyclooctadiene lignans in agar cultures and in stationary and agitated liquid cultures of Schisandra chinensis (Turcz.) Baill. Appl Microbiol Biotechnol 100:3965-3977. https://doi.org/10.1007/s00253-015-7230-9

Szopa A, Ekiert R, Ekiert H (2017a) Current knowledge of Schisandra chinensis (Turcz.) Baill. (Chinese magnolia vine) as a medicinal plant species: a review on the bioactive components, pharmacological properties, analytical and biotechnological studies. Phytochem Rev 16:195-218. https://doi.org/10.1007/ s11101-016-9470-4

Szopa A, Kokotkiewicz A, Luczkiewicz M, Ekiert H (2017b) Schisandra lignans production regulated by different bioreactor type. J Biotechnol 247:11-17. https://doi.org/10.1016/j.jbiot ec.2017.02.007

Szopa A, Dziurka M, Warzecha A et al (2018a) Targeted lignan profiling and anti-inflammatory properties of Schisandra rubriflora and Schisandra chinensis extracts. Molecules 23:3103. https:// doi.org/10.3390/molecules23123103

Szopa A, Klimek-Szczykutowicz M, Kokotkiewicz A et al (2018b) Phytochemical and biotechnological studies on Schisandra chinensis cultivar Sadova No. 1-a high utility medicinal plant. Appl Microbiol Biotechnol 102:5105-5120. https://doi. org/10.1007/s00253-018-8981-x

Szopa A, Barnaś M, Ekiert H (2019a) Phytochemical studies and biological activity of three Chinese Schisandra species (Schisandra sphenanthera, Schisandra henryi and Schisandra rubriflora): current findings and future applications. Phytochem Rev 18:109-128. https://doi.org/10.1007/s11101-018-9582-0

Szopa A, Klimek-Szczykutowicz M, Kokotkiewicz A et al (2019b) Phenolic acid and flavonoid production in agar, agitated and bioreactor-grown microshoot cultures of Schisandra chinensis cv. Sadova No. 1-a valuable medicinal plant. J Biotechnol 305:61-70. https://doi.org/10.1016/j.jbiotec.2019.08.021

Szopa A, Kokotkiewicz A, Bednarz M et al (2019c) Testing production of phenolic compounds-phenolic acids and flavonoids in microshoots cultures of Schisandra chinensis (Turcz.) Baill. cultivated in different plant bioreactors. Plant Cell Tissue Organ Cult 139:199-206. https://doi.org/10.1007/s11240-019-01676-6
Szopa A, Kokotkiewicz A, Klimek-Szczykutowicz M et al (2020) Different types of in vitro cultures of Schisandra chinensis and its cultivar ( $S$. chinensis cv. Sadova): a rich potential source of specific lignans and phenolic compounds. In: Ramawat $\mathrm{K}$, Ekiert H, Goyal S (eds) Plant cell and tissue differentiation and secondary metabolites. Reference series in phytochemistry. Springer, Cham, pp 1-28

Taguchi H, Ikeya Y (1975) The constituents of Schizandra chinensis Baill. I. The structures of gomisin A, B and C. Chem Pharm Bull 23:3296-3298. https://doi.org/10.1248/cpb.23.3296

Upton R, Graff A, Jolliffe G et al (2011) American herbal pharmacopoeia: botanical pharmacognosy-microscopic characterization of botanical medicines. CRC Press, Boca Raton

Verpoorte R, Contin A, Memelink J (2002) Biotechnology for the production of plant secondary metabolites. Phytochem Rev 1:13-25. https://doi.org/10.1023/A:1015871916833

Wang YH, Qiu C, Wang DW et al (2011) Identification of multiple constituents in the traditional Chinese medicine formula ShengMai San and rat plasma after oral administration by HPLCDAD-MS/MS. J Pharm Biomed Anal 54:1110-1127. https://doi. org/10.1016/j.jpba.2010.11.034

Wiart C (2013) Lead compounds from medicinal plants for the treatment of cancer. Elsevier Academic Press, London

World Health Organization (2007) WHO Monographs on Selected Medicinal Plants. Fructus Schisandrae, vol 3. WHO, Geneva

Yang JM, Siu-po P, Hok-keung JY, Che C (2011) HPLC-MS analysis of Schisandra lignans and their metabolites in Caco-2 cell monolayer and rat everted gut sac models and in rat plasma. Acta Pharm Sin B 1:46-55. https://doi.org/10.1016/j.apsb.2011.04.007

Zabala MA, Angarita M, Restrepo JM et al (2010) Elicitation with methyl-jasmonate stimulates peruvoside production in cell suspension cultures of Thevetia peruviana. In Vitro Cell Dev Biol Plant 46:233-238. https://doi.org/10.1007/s11627-009-9249-Z

Zhu M, Chen XS, Wang KX (2007) Variation of the lignan content of Schisandra Chinensis (Turcz.) Baill. and Schisandra sphenanthera Rehd. et Wils. Chromatographia 66:125-128. https://doi. org/10.1365/s10337-007-0261-3

Zou D, Wang J, Zhang B et al (2015) Analysis of chemical constituents in Wuzi-Yanzong-Wan by UPLC-ESI-LTQ-Orbitrap-MS. Molecules 20:21373-21404. https://doi.org/10.3390/molecules2 01219765

Publisher's Note Springer Nature remains neutral with regard to jurisdictional claims in published maps and institutional affiliations. 